\title{
4. Fremde Einflüsse bei der Entwicklung des Usinšs
}

4.1. Das analysierte Quellenmaterial ermöglicht die Bestimmung des Wesens des Ūsinšs. Es ist nun möglich, sich kritisch zu den bisherigen Ansichten zu äußern, die, wie schon erwähnt, bis in die jüngste Zeit sehr unterschiedlich und sogar scharf widersprüchlich waren. Es liegt auf der Hand, daß man beim Aufzeigen der Mängel der bisherigen Anschauungen einen gewissen Negativismus nicht vermeiden kann. Wenn hier Anschauungen genannt und kritisiert werden, die offensichtlich irrtümlich sind, dann geschieht das, weil solche noch immer auch in bekannten Arbeiten anzutreffen sind.

4.1.1. Den Forderungen und Traditionen jener Zeit entsprechend, stützten sich die ersten Versuche bei der Bestimmung des Wesens des Ūsiňš auf die Etymologie des Namens. Auniņš 1881 veröffentlichte Arbeit kann man als ersten derartigen Versuch ansehen. Diese bietet zwar ein sehr umfangreiches folkloristisches Material, beruft sich aber bei der Erklärung des Namens Üsinš ausschließlich auf etymologische Zusammenhänge. Er verbindet unter Berufung auf die Äußerungen der Linguisten seiner Zeit den Namen Úsinš mit der ide. Wurzel us-1. An dieser seiner Anschauung hielt er bis an sein Lebensende fest². Dabei ist aber sofort anzumerken, daß er keine genaue linguistische Analyse bietet, die den Namen Ūsiņš eindeutig mit der erwähnten Wurzel verbindet. Dazu haben ihn einerseits die Äußerungen allgemeiner Natur von Bezzenberger und Bielenstein veranlaßt3, andererseits die fonetische Ähnlichkeit, die bei den Wörtern Ușas und Ūsinšs wahrzunehmen ist. Es liegt auf der Hand, daß Endzelins, der tieferen Einblick in die altindische Sprache hatte, die Verbindung des Namens Usinšs mit dieser Wurzel nicht akzeptieren konnte. Er sagte deklarativ: „,Daß aber der Name Üsinšs nicht von der Wurzel aus-, die in den Wörtern

1 Cf. Aunings 1881, 39 ss; auch id. 1905, 30.

2 So schreibt er 1913, 27: ,Ich wiederhole, was ich bereits früher an einer anderen Stelle gesagt habe: Es ist hochinteressant, daß von der Einen uralten Wortwurzel us (vas) eine ganze Reihe von Lichtgottheiten der Arier (Indogermanen) ihren Namen haben."

${ }^{3}$ Cf. Auniṇš 1881, 39. 
'austra' - Morgenröte -, 'austrums' - Osten -, steckt, abzuleiten, sondern als Entlehnung aufzufassen ist, daran halte ich auch jetzt fest. "1 Diese kritischen Worte von Endzelīns aber fanden keinerlei Widerhall bei den Religionsforschern jener Zeit. Die Anschauung, daß der Name Ūsiņs mit der Wurzel $u s$ - zu verbinden sei, ist bis auf den heutigen Tag anerkannt ${ }^{2}$. Das ist verständlich, weil das auch in der Arbeit des so sehr bedeutenden Forschers Schröder eine Stütze fand ${ }^{3}$. So wurde er dann auf rein etymologischer Basis mit dem altindischen uṣas verbunden.

In jüngster Zeit hat sich mit der Etymologie des Wortes Schmid befaßt und in die Angelegenheit weitgehende Klarheit gebracht: ,,Die vermeintliche etymologische Verwandtschaft von $\breve{U}$ siņš und usás- läßt sich jedoch Punkt für Punkt als falsch erweisen. Da es sich bei diesem Vergleich um eine Wurzeletymologie handelt, bei der die unterschiedliche Stammbildung und damit das verschiedene Genus beider Wörter nicht berücksichtigt wird, muß man verlangen, daß die Verbindung $\bar{u} s$ - mit altind. us- tadellos ist. Tatsächlich aber stimmt weder das $u$ noch das $s$. Wie gerade das Altindische zeigt, hat die Schwundstufe der Wz. *aues- stets kurzes -u-. Altind. uṣás- == avest. us̆ah- ,Morgenröte" ", altind. ucchati=avest. usaiti ,leuchtet auf", usrá- „morgendlich" "[1]. $\bar{U}$ siňs hat aber stets langes $\bar{u}$. Man könnte sich allerdings auf das žemaitische apýūšriai ,Morgendämmerung" berufen $^{[2]}$, das aber nicht nur ausnahmsloser Stufe -au- im Baltischen gegenübersteht, sondern auch schriftlitauisches apýaušris zur Seite hat, so daß diese nicht eindeutige Form ${ }^{[3]}$ kein zweifelsfreier Zeuge für einen Ablaut indogerm. Alters darstellt. Will man jedoch der Quantitätsdifferenz $\bar{u} / u$ keine Beweiskraft zumessen, dann wird die slav. Form ycéнь falsch; denn geht man von altem $-u$-oder $-u$-Diphthongen aus, muß das $s$ nach $\breve{u}$ zu slav. - $x$ - werden, vgl. russ. ýxá „Fischsuppe", lit. júšé ,Fischsuppe", altind. $y \bar{u} s a^{a} n-$,Brühe", oder russ. ýxo ,Ohr": lit. ausis, lat. auris ,,Ohr"; russ. сухой ,trocken": lit. saũsas, ,dass.", u.a.m. Also: nach * slav. $s$ regelwidrig; will man aber an dem $s$ festhalten - wie in russ. yc, lett. $\vec{u} s a s$,Schnurrbart" hier bereits russ.-ksl. Qsz ,Schnurrbart", apreuß. wanso ,der erste Bart" zeigen. Daraus ergibt sich, daß allein aus lautlichen Gründen weder üsings noch усе́нь zu altind. usás- bzw. zur Wz. *aues- gehören kann. Es kommt hinzu, daß auch lett. $\bar{u}$ und russ. $y$ nicht zueinander passen, wenn man von $\breve{u}$

1 Endzelins 1908, 134.

2 Cf. Brastiņš 1956, 75; Audriņš $34 \mathrm{~s}$.

a Schröder 2,51 s: ,,Schon der Name spricht deutlich für die Annahme, denn unzweifelhaft richtig hat Auniṇš denselben von der Wurzel vas, us abgeleitet, die 'aufleuchten, brennen' bedeutet und so vielen arischen Namen der aufleuchtenden Sonne, der Morgenröte, wie auch verschiedener. Sonnengötter und Sonnenheroen zugrunde liegt." 
ausgeht. - Der Verweis auf RV. VII 77, 5 ist alles andere als stichhaltig, denn die Bitte um gómad áśvāvad ráthavac ca rádhah steht auch RV. V 57, 7, dort an die Maruts gerichtet. Auch Indra schenkt z. B. Rosse und Rinder (VIII 6, 24; 12, 33). Selbst weitere Vergleiche, die sich zwischen Usásund $\bar{U} \operatorname{sings~herstellen~ließen,~sind~sämtlich~nicht~typisch.~Beide~nahen~z.~B.~}$ mit Pferd oder Pferdegespann, - doch das tut auch Indra; beiden wird in der Frühe geopfert, - doch auch Agni empfängt Opfer am Morgen. Das Wohnen bzw. Sitzen auf dem Berg ist ein naturalistischer Zug, der im RV. auch von den Aśvins ausgesagt wird. Es gibt also weder grammatische noch inhaltliche Besonderheiten, die zwingend für eine Gleichsetzung von $\bar{U}$ sinš und usás- sprechen. Die lautliche Form der Namen entscheidet vielmehr dagegen."1 Wir verstehen nun die von Endzelīns geäußerten Bedenken gegenüber der Verbindung von $\bar{U}$ siņš mit der Wurzel us- besser. Diese lange Zeit behauptete etymologische Beziehung kann hinfort nicht mehr vertreten werden. Die Ablehnung der etymologischen Beziehungen hat auch Konsequenzen inhaltlicher Art. Auf Grund der vermuteten etymologischen Beziehungen wurde Ūsiņš als Gott mit anderen von dieser Wurzel abgeleiteten indoeuropäischen Götternamen bzw. Göttern identifiziert. Besonders beliebt war seit der Zeit von Auniṇš und Schröder die Berufung auf uṣas. Wir erinnern hier nur an des letzteren Worte: , Ein Gott der aufleuchtenden, aufsteigenden Sonne war aller Wahrscheinlichkeit nach der lettische Uh sing wie die indische Ushas, Eos, die germanische Ostara diese Naturerscheinung in weiblicher Form repräsentieren, der indische Vivasvant in männlicher."2 Diese Behauptung findet keine Stütze an der Linguistik und deshalb entfällt eine derartige Motivierung für die Identität des Ūsiņš mit ähnlichen indoeuropäischen Göttern ${ }^{3}$. Daß diese irrtümliche Anschauung sich so lange

1 Schmid 134 s. Er hat seinem hier erwähnten Zitat die folgenden Anmerkungen beigefügt: ,,l. Vgl. Renou 4, Anm. 2; 2. Trautm ann19; Pokorny 87. Das $\bar{u}$ von klass. sanskrit pratyüșa-= prabhāta- ,Tagesanbruch" hat keinen Anspruch auf Altertümlichkeit; s. Renou 5; zur Flexion von uṣás- vgl. Wackernagel 3, 281 ff; Manessy 20 ff; 3. Es könnte sich z. B. auch um einen Ablaut handeln, wie er in lit. dìbti ,,sich höhlen", dúabti ,aushöhlen", dauburỹs, dŭburys „Wasserloch", duobẽ „,Grube, Loch“ vorliegt. uo wird im Südost.Žemaitischen korrekt zu $\bar{u}$ (siehe Būga 3,94)." Diese Worte von Schmid über den Zusammenhang von $\overline{u s a s ~ m i t ~ d e m ~ r u s s i s c h e n ~ y c e н ъ ~ d e c k e n ~ s i c h ~ m i t ~}$ der Behauptung von Endzelins: „Demnach kann aus der Wurzel aus- nur 'У entstehen, nicht aber 'Усень'. [...] Das ursprüngliche au hat sich in den slavischen Sprachen zu $u$ geändert (ursprüngliches $u h$ dagegen zu $b l$ z. B. dūmi: дымъ. Denmach ist der Wurzelvokal in den Wörtern 'Uhsinssch' und 'Усень' nicht identisch: an der Stelle des russischen y müßte lettisch au stehen und an der Stelle des lettischen $u h$ das russische bा $(1908,134)$.

2 Schröder 2,52.

3 Über die im Rigv auftretenden Göttinnen Ușas und den entsprechenden Wesen in der Religion der Balten habe ich mich in anderem Zusammenhang genauer geäußert. Cf. Biezais, 1972, $285 \mathrm{ss}, 292 \mathrm{~s}$. 
hat halten können, ist aus mehreren Umständen zu erklären. Vor allem: sie war von den anerkannten Linguisten und Religionswissenschaftler jener Zeit ausgesprochen worden. Weiter: sie hatte schon sehr früh bei den lettischen Forschern Anklang gefunden. Schließlich: sie entsprach den nationalromantischen Tendenzen jener Zeit, die Beziehungen zwischen der altlettischen und der altindischen Religion nachzuweisen versuchten ${ }^{1}$.

4.1.2. Seit den Zeiten Stenders ist eine Erklärung des Wesens von Ūsiṇš bekannt, die sich auf eine andere Etymologie dieses Wortes stützt. Das Wort sei in diesem Falle mit $z$ zu schreiben und zu sprechen: $\vec{U}_{z i n z s}$. Er nahm an, daß der Name auf das Lehnwort $\bar{u} z a s$ 'Hosen' zurückzuführen sei. Es sei wegen der gelben „Hosen“ der Bienen entstanden, denn Ūziņš sei doch der Gott der Bienen. Die gelehrte Tradition hat dann diese Anschauung bis in unsere Tage hinübergetragen. Wir haben schon in der Quellenanalyse darauf hingewiesen, daß in älterem Folklorematerial keinerlei Hinweis auf einen Gott anzutreffen sei, der als $\bar{U}$ zainis, der 'Behoste', bezeichnet wird. Nur in jüngeren Dainas-Texten unseres Jhs. die eindeutig die Freude am

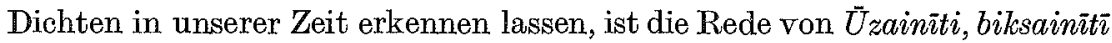
'Behoster' Voc. $(72,73)$ Dieser unter dem Einfluß von Diskussionen und Publikationen gelehrter Leute entstandener Tradition ist bei der Erörterung des Ūsiņš-Problems keinerlei Bedeutung beizumessen. Es muß freilich vermerkt werden, daß auch in der älteren lexikalischen Tradition ein gewisses Schwanken in der Schreibung des Namens Üsiňš zu beobachten ist, was aber, wie wir gesehen haben, auf die instabile lettische Rechtschreibung bzw. auf mundartliche Erscheinungen zurückzuführen ist. Der Pseudogott Ūzainis wird dann in dieser Tradition als Bienengott aufgefaßt und Ūziņš genannt. Die zugängliche Quelle, eine einzige Daina (74) gehört in eine sehr späte Zeit und ist als freie Dichtung anzusehen. Der Verbreiter dieser Tradition war Tortuss, der dann auch angeblich im Volke vernommenes Material über die Beziehung zwischen Ūsiṇš und den Bienen bringt. Derartiges Material ist fast ausschließlich bei ihm zu finden ${ }^{2}$. Außer mit dem von ihm gebrachten Folklorematerial begründet Tortuss die Unterscheidung des Pferde- und des Bienengottes mit den angeblich von ihm gehörten unterschiedlichen Götternamen Ūsiņš und Uziņs. Das entbehrt, wie wir gesehen haben, jeder Grundlage. Seinerzeit hat auch Endzelins von Usiņš als vom Bienengott gesprochen, wobei er sich offenbar, denn auf anderes beruft er sich nicht, allein auf lexikalische Angaben stützt. Man ersieht das auch aus seinem Vorwurf gegenüber Auninšs, denn ,,wie der auch zum Bienengöttchen geworden ist, erklärt auch Auniņš nicht“'3. Aus dem oben Gesagten geht

1 Über die Tendenzen Biezais, 1961, 44 ss.

2 Cf. Ozolin̄̌s 103 s; Pasakas 1893 s, 365, 368.

3 Endzelins 1908, 135. 
hervor, daß auch der Vorwurf von Endzelīns unbegründet ist. Wenn man unbedingt die sekundäre Tradition, woron hier die Rede ist, beachten und eine Erklärung für die Verknüpfung des Usinšš mit den Bienen bringen will, könnte man mit derselben spekulativen Antwort aufwarten, die Auniņš dem Endzelīns gegenben hat. Auniņš verweist zuerst auf das 36. Lied und fährt dann fort: ,,Derselbe Uhsin, der den Pferden das frische Grün bringt, bringt auch den Bienen die schönen Knospen und Blumen, wo sie den süßen Honig finden können. "1 Das bedeutet, daß Auniṇš ganz richtig erkannt hat, daß die Bedeutung des Ūsiņš über das Patronat über die Pferde hinausreicht, wie das auch mehrere der von uns erörterten Texte ausweisen. So konnte erlebnisgemäß Ūsiņš auch mit den Bienen in Verbindung gebracht werden, ohne daß er deshalb zum speziellen Bienengott zu werden brauchte. Zugleich aber ist zu sagen, daß die von Stender eingeführte Tradition vom behosten Bienengott, der im späteren Folklorematerial dann Uzainis genannt wird, ein auf Grund von Volksetymologie entstandener Pseudogott ist.

4.1.3. Viel verwickelter ist die Frage nach dem in unseren Texten anzutreffenden $\bar{U}_{s a}$ bzw. $\bar{U} s s$ (33) und Üsainitis (46 c). Die erste, ,in den Volksliedern einmal auftretende Form Uss ist als Abstraktion von der anscheinenden Deminutivform $\bar{U}$ siňs anzusehen, die in Wahrheit durchaus kein Deminutiv ist, sondern die ursprüngliche Form dieses Namens ${ }^{* 2}$. Die erste Hälfte dieser Behauptung von Endzelins ist zweifelsohne richtig, und $\bar{U}_{s s}$ ist als abgeleitete Bildung von Ūsiņ̌s anzusehen, während die zweite Hälfte der Behauptung auf den Abweg geführt hat, den Namen Ūsinšs auf Entlehnung aus dem Deutschen oder dem Russischen zurückzuführen. Schließlich waren Endzelīns und sein Gefolgsmann Smits gezwungen, zu der Behauptung zurückzukehren, die schon Müthel ausgesprochen hatte, daß nämlich der Name des Gottes Ũsiņš auf die Vorstellung von üsas 'Schnurrbart' zurückzuführen sei. Die im Text 46 anzutreffende Form bestätigt zweifelsfrei die Ableitung von ūsas. Das könnte dann eine Begründung für die Behauptung von Endzelins sein, auf diese Weise sei der Name Üsinš entstanden. Es ist aber anzumerken, daB der Text 46, dessen Varianten mit Úsainitis 'der Schnurrbärtige' dem Text 30053 entsprechen, sehr spät, nämlich erst im 20. Jh. aufgeschrieben wurde, und dazu diese Form sich ausschließlich in den Varianten dieses Liedes findet. Weiter fällt in einem Teil dieser Varianten eine große Unsicherheit auf: es finden sich darin Ussainitis, Usenitis, Usinitis, Usitis, was zur Annahme von Instabilität bei diesem Text veranlaßt. Schließlich wird diese Bezeichnung wechselweise mit Jurǵztis, 'hl. Georg', verwendet. Sieht man endlich die Varianten des Liedes $30053 \mathrm{im}$ Lichte von Text 46 bei Auniņš, in dem sich die Form Ūsiņš findet,

1 Auniņs 1913, 18.

2 Endzelīns 1903, 25. 
wird deutlich, daß 30053 in Wahrheit eine Nachdichtung dieses Liedes ist. Das sind Umstände, die begründete Zweifel an dem schnurrbärtigen Gott Ūsinšr rechtfertigen. Schon Müthel war sich dessen bewußt, daß um vom schnurrbärtigen Gott reden zu dürfen, in den religiösen Vorstellungen des Volkes irgendwelche Grundlagen dafür vorhanden sein müßten: „Falls die alten Letten sich den Uhsinssch als ihren Pferdegott unter dem Bilde eines gewaltigen Reiters gedacht haben, der unter anderen Eigenschaften auch mit einem Schnauzbart geziert gewesen sein mag, so scheint mir sein Name von Uhsa (uhsas — Schnurrbart) abgeleitet werden zu können und würde soviel bedeuten als uhsains (der Schnurrbärtige). "1 Die Nachfolger Müthels, oder richtiger, die Erneuerer seiner Gedanken, Endzelins und Smits, haben das kritiklos übernommen. Müthel hat sich durchaus nur hypothetisch ausgedrückt: ,Falls [...], so scheint [...]." Mit anderen Worten, er war sich, als er seine Hypothese aussprach, dessen bewußt, daß sie der Verifizierung bedurfte. Er selbst hat eine solche nicht gebracht, hat sie aber von denen erwartet, die seine Anschauungen überprüfen. Diese Hypothese ist von seinen beiden Nachfolgern ohne jede weitere Begründung als richtig angesehen worden. Schon aus rein formalen wissenschaftstheoretischen Gründen ist diese Hypothese als unbewiesen anzusehen. Auniņš hat sie zu seiner Zeit abgelehnt, aber auch er nennt keine Gründe für die Ablehnung ${ }^{2}$. $\mathrm{Zu}$ dieser Frage werden wir nochmals zurückkehren, wenn wir uns mit dem EinfluB fremder Religionen, insbesondere dem vom hl. Georg, auf die Gestalt des Ũsiņš bekanntgemacht haben.

4.2. Es versteht sich von selbst, daß, da die Letten in Berührung mit anderen Völkern gelebt haben, Elemente der Sprachen dieser Völker in die lettische Sprache eingedrungen sind. Ebenso mußte es mit religiösen Vorstellungen geschehen. Es erübrigt sich, an dieser Stelle genauer auf die Gestaltungsvorgänge des Synkretismus einzugehen ${ }^{3}$. Einen solchen kann man erwarten, und ein solcher wurde auch in Bezug auf Üsinš vermutet. Derarige Vermutungen können wir jetzt im Lichte unserer Quellen überprüfen.

4.2.1. Das Erforschen des Usiņ̌s-Problems ist heute soweit fortgeschritten, daß es mit völliger. Sicherheit möglich ist, aus der zukünftigen Diskussion die Anschauungen auszuschließen, die im Ūsinšs die Entlehnung des in der deutschen Volksüberlieferung bekannten Husing sehen wollen. Offensichtlich ist Endzelīns zu jener Anschauung weniger durch die mit dem deutschen

1 Auniņ̌ 1881, 10.

2 Ib.: „Die von ihm [Müthel] proponierte Ableitung des Namens Uhsiņsch halten wir für ebenso unrichtig wie die von Stender in seiner Grammatik pag. 271 gegebene."

${ }^{3}$ Cf. den in der gleichen Reihe erschienenen Spezialband "Syncretism", sowie Biezais 1976. 
Husing verbundenen religiösen Vorstellung, die keinesfalls mit denen übereinstimmen, die mit Ūsinš verknüpft sind, veranlaßt worden, als vielmehr durch die wahrzunehmende fonetische Ähnlichkeit der beiden Namen. Später war auch Endzelīns gezwungen, seine Anschauung zu widerrufen und die Forschung wird, in Zukunft keine Veranlassung haben, zu ihr zurückzukehren.

4.2.2. Viel energischer ist die Anschauung vertreten worden, nach der Name und Erscheinungsbild des Ūsiņš eine Entlehnung aus dem russischen Kulturkreis sei, wobei er mit У сень verknüpft wird. Das ist darauf zurückzuführen, daß diese Anschauung von zwei Seiten verteidigt wurde. Einerseits haben sich russische Forscher auf lettische, insbesondere auf Auniņ̌ berufen, andererseits lettische, und dort, allen voran, wieder Endzelins, auf russische. Darüber habe ich mich schon früher geäußert'1. Fragt man nach der Begründung dieser Anschauungen, ergeben sich mehrere Schwierigkeiten. Vor allem ist bis heute die Bedeutung des russischen Wortes nicht eindeutig geklärt. Sie schwankt zwischen einem Liederrefrain ohne semantisch bestimmbare Bedeutung bis zu ,der erste Tag des Frühlings " und ,,ein am Sylvesterabend vor den Fenstern mißliebiger Personen im Dorfe gesungenes Spottlied“. Sicher ist jedenfalls, daß die russische Volksüberlieferung kein religiöses Wesen namens У сень kennt. Rein hypothetisch schließt das gewiß die Möglichkeit der Übernahme des Wortes selbst nicht aus, welches dann in der lettischen religiösen Überlieferung einen anderen Sinngehalt erhalten hätte. Aber auch in diesem Falle bleibt unbestreitbar, daß Ūsiņš als personifiziertes Wesen nicht unter dem Einfluß des russischen Wortes entstanden, noch auch entlehnt worden ist. Wollte man sich noch weiter in derartige Spekulationen einlassen, die freilich nur Vermutungen zu unserer obigen Diskussion wären, könnte man meinen, dieses Wesen sei aus der russischen Tradition geschwunden, von der lettischen aber übernommen und darin bewahrt worden. Das aber ist und bliebe nur eine lose unbegründete Spekulation. Diese Einsicht befreit uns freilich nicht von der Pflicht, zu klären, wie Endzelins und die erwähnten russischen Forscher zu dieser Anschauung gelangt sind. Die Entstehung dieser Anschauung hat Auniñš schicksalshaft bestimmt. Er beruft sich auf Bezzenberger, der den Namen Ūsiņš etymologisch mit us- und damit mit einer Reihe bei indoeuropäischen Völkern bekannter Götter, deren Namen von dieser Wurzel abgeleitet sind, verbindet. Diese etymologische Beziehung übernahm auch dann vor allem Potebnja und, wie wir sahen, seine Nachfolger. Auch Volteris schloB sich ihm an. Diese ganze Forschergruppe hat sich stärkstens von der phonetischen Ähnlichkeit des lettischen und des russischen Wortes leiten

\footnotetext{
1 Ci. oben S. 16 ss.
} 
lassen und von deren anscheinendem Zusammenhang mit der dem indoeuropäischen Sprachen gemeinsamen Wurzel.

$\mathrm{Zu}$ einem mehr anfechtbarem, ja unverständlichen Standpunkt ist Endzelīns gelangt. Wie wir wissen, verweist er nachdrücklich auf den seit Bezzenberger behaupteten und von lettischen und russischen Forschern übernommenen etymologischen Zusammenhang zwischen den Wörtern Ūsiņš, Усень und der indoeuropäischen Wurzel us-1. Woher nimmt er dann die Möglichkeit, von einer Entlehnung des Ūsiņš aus dem Russischen zu sprechen? Er antwortet: „Man mag den russischen Усень oder Овсень so oder so erklären; daran ist aber wohl nicht zu zweifeln, daß die Letten ihren Ūsiņš von den Russen entlehnt haben. “a Obwohl er den lautlichen Zusammenhang nicht Beweisen kann, spricht er von Entlehnung. Danach kann man nur noch an eine Entlehnung des Wesens von Ūsiņ̌s denken, die vom Namen unabhängig ist. Wie aber eben gesagt, kennen die Russen kein Wesen, das dem lettischen Ūsiňš entspräche. Deshalb sind die seit den Zeiten von Auningš bestehenden Spekulationen über Verbindungen des Wortes Ūsiņš mit phonetisch ähnlichen russischen Wörtern, oder gar über die direkte Entlehnung dieses Wesens abzulehnen. Hier ist an die obenerwähnte eingehende und überzeugende Analyse von Schmid zu erinnern ${ }^{3}$. Ungeachtet dessen, daß, wie Vasmer sagt, ,dieses lautlich unmöglich ist", könnte ein spekulativ gestimmter Forscher einwenden, die lebendige Sprache kümmere sich nicht um die formalen Gesetze sprachlicher Bildungen der Philologen und dies sei eine spontane Sprachäußerung. Derartige Spekulationen aber sind unfruchtbar und unverbindlich.

4.2.3. Wir haben schon bei der Erörterung der Quellen angemerkt, daß sie alle dem christlichen Zeitabschnitt im Leben des lettischen Volkes angehören. Schon diese Feststellung ist ein formal ausreichender Grund für die Annahme, das Wesen des Ŭsiņš sei durch die christliche Tradition mitbestimmt. Dabei ist nur daran zu erinnern, daß die Nachrichten sich in der Meldung des jesuitischen Priesters und Missionars Stribiņš vom Jahre 1606 finden. Da liegt das Motiv dafür, daß in unserer Erörterung des Problems der Einfluß der christlichen Kirche auf Ūsinšs besondere Aufmerksamkeit verdient.

Mehrfach begegneten wir sowohl im lexikalischen wie im Folklorematerial der Erwähnung des Ūsiņtages, wobei gewöhnlich die Pluralform des Wortes Ūsinš, nämlich $\bar{U} \operatorname{sini} i$ benutzt wird. Bei Ereignissen, die direkt mit diesem Tage verbunden sind, etwa dem Mahl, benutzt man als Zeitbestimmung dieses Wort im Lokativ der Mehrzahl Ūsinos. In genau der gleichen Bedeu-

1 Cf. Endzelins 1908, 134.

2 Ib.

3 Cf. oben S. 124 s. Dies hat seinerzeit auch Vasmer 1,3 s; 3, 190 abgelehnt. 
tung und im gleichen Zusammenhang benutzt man den Namen Georg aus dem christlichen Kalender, freilich nur in den im Volke üblichen abgewandelten Formen Juris, Jurǵis ${ }^{1}$, aber am häufigsten in den Deminutivformen Jurūtis, Jurğtis, sogar in der offensichtlichen Übergangsform von Georg zu Juritis, nämlich Gurǵitis $(30057,2)$. Der U̦siņtag war, wie der Georgstag, der 23. April. Als Bezeichnung für diesen Tag sind beide Wörter im Volke tief verwurzelt, das hat 1772 schon Lange ganz richtig vermerkt ${ }^{2}$. Als Zeitbestimmung werden beide Wörter synonym verwendet. Es ist nicht möglich, diese Identität der Bezeichnung in einen früheren Zeitraum, als den des Erscheinungsjahres von Langes Wörterbuch, also 1772, zu verlegen. Es besteht aber kein Anlaß daran zu zweifeln, daß die Tradition ihre Entstehungszeit hatte, darüber aber fehlen Nachrichten.

Über die Identifizierung des Üsintages mit dem Tag des hl. Georg hat sich Adamovičs geäußert. Nachdem er alle uns bekannten mit dem Úsinšstag verbundenen Traditionen kurz referiert hat, folgert er: ,Den Ablauf aller dieser Tätigkeiten und Riten darf man sich nicht als auf einen Tag konzentriert vorstellen, er erfolgte hingegen in einem ganzen Zeitraum, sagen wir, dem Laub- und Saatmonat, wobei vielleicht der Anfangstag besonders hervorgehoben wurde. Der Ūsinštag konnte, als es noch keinen eigenen Kalender gab, auf keinem bestimmten Datum liegen und ist vielleicht ein später eingedrungenes Wort. Später identifizierte man ihn mit Georgi."3 $\mathrm{Zu}$ diesen Worten des Adamovičs ist wohl nur der Klarheit willen anzumerken, daB er unter dem Laub- und Saatmonat den Mai versteht ${ }^{4}$. Beachtet man, daß die alten Letten keinen ausgearbeiteten Tageskalender hatten, oder besser, daß ein solcher nicht bekannt ist, besteht kein Grund,

1 Es ist bezeichnend, daß auch diese beiden in der Volkssprache vorkommenden Formen die Kirche offiziell sanktioniert und in den Kalender aufgenommen sind. Cf. Grāmata 1975, 12. Um aber denen entgegenzukommen, die ihrem Kinde den Namen des vorchristilichen Gottes Ūsiņš gegeben haben, hat man, ohne die Usinšstraditionen zu kennen, ihm einen falschen Tag, nämlich den 22. April zugewiesen (of. Grämata 1952, 6; Kalendars 1975, 15).

${ }^{2}$ Cf. Lange 363; auch Arājs 1957, 635 und 1957a, 800; LE 1, 918, Greble, Vilma 1969, 217; Ozols 1955, 432; Zeiferts 1957, 1, 237.

${ }^{3}$ Adamovičs 95. Eine ähnliche Erscheinung ist auch bei anderen Völkern nach Einführung des christlichen Kalenders festzustellen. So sagt Rosen über Balkanvölken (Volkslieder 24) bei der Zuweisung von Liedern verschiedenen Inhalts zum Kalender: ,Diese Einteilung, wenn auch durch Übung und Überlieferung geheiligt, beruht keineswegs auf innerer Notwendigkeit, d. h. der Inhalt der Lieder steht in der Regel auch nicht in der allerentferntesten Beziehung zu dem Kalendertage, dessen Namen sie tragen, oder zu dem Anlaß, bei dem sie gesungen werden." Cf. auch Serben $239 \mathrm{~s}$; Reuter $12 \mathrm{ss}$.

4 Der Name Laub- oder Saatmonat ist sehr alt, man findet ihn schon bei Einhorn 1649 (Einhorn 1649, 23). Diese alte Bezeichnung für den Mai hat der offizielle katholische Kalender bis in unsere Tage erhalten (of. Kalendārs 1974, 12). 
von einem bestimmten Tag als dem Ūsinšstag zu sprechen. Daraus folgert dann auch Adamovičs, daß die Identifizierung des Ūsiņštages mit Georgi ein Vorgang aus späterer Zeit sei. In diesem Zusammenhang hat auch er sich unsicher ausgedrückt, wenn er sagt, daß Ūiñš ein ,,vielleicht später eingedrungenes Wort" sei. Darüber aber wird später zu reden sein. Es ist jedenfalls festzuhalten, daß beide Bezeichnungen durch mehrere Jhe. und bis in unsere Zeit nebeneinander benutzt worden sind. Diese Einsicht wirft eine Reihe weiterer Fragen auf. Bevor wir aber auf diese eingehen, wollen wir betrachten, welche Angaben uns die Quellen in Bezug auf die religiösen Vorstellungen der Letten über Ūsiņš und den hl. Georg bieten.

4.2.3.1. Das Tätigwerden von Uিsinš und dem hl. Georg in den gleichen Funktionen ist in den Texten mehrfach bezeugt. Sie werden oft genug nebeneinander genannt. Wir haben schon früher den Text 46 kennengelernt, in dessen erster Zeile Ūsiņš mit der Apposition ,guter Mann" angeredet wird. Dieses Iied hat schon Auniñš aufgeschrieben. In dem LD aber finden wir den folgenden Text (46b):

Ai, Jurginti, ai, $\bar{U} s \bar{t} t i$,

Baro labus kumelinus!

Sukādams, barodams,

Sēsties siles galinā.
Ei, Jurǵiti, ei, Ūsīti, füttere gutø Rosse dim. indem [du] sie fütterst, striegelst, setz dich ans Ende dim. des Futtertrogs.

In diesem Text ist die 1. Zeile insofern verändert, als der hl. Georg an erster Stelle genannt wird und neben ihm auch Ūsitis bzw. Üsiņš. Wichtiger a ber als die Nennung der Namen nebeneinander ist deren Verbindung mit den gleichen Funktionen - das Füttern und Striegeln der Pferde und schließlich die Aufforderung, sich auf den Ehrenplatz am Ende des Troges zu setzen. Eine andere Variante $(46 \mathrm{c})$ desselben Liedes geht noch weiter und nennt den hl. Georg selbst $\breve{U}$ sainīti: $A i$, Jurgiti $\bar{U}$ sainīti. Formal ermöglicht der Text die Meinung, man habe sich den hl. Georg schnurrbärtig vorgestellt. Diese Meinung entbehrt gewiß der Grundlage. Offensichtlich wird der hl. Georg auf Grund von Kontamination Usainitis genannt. Das auf ihn bezogene Attribut gehört in Wahrheit zu Ūsinšs, was wir in der vorhergehenden Erörterung schon gesehen haben. Diese Kontamination wird auch durch den Inhalt des Textes bestätigt, da ja beide in der gleichen Funktion auftreten. Die Vermischung von Ūsinš und dem hl. Georg zeigt, daß zur Zeit der Aufzeichnung der Iieder zwischen beiden keine Grenze mehr zu ziehen war. Das hat der dichterischen Phantasie weiteren Spielraum eröffnet. In anderen Varianten desselben Liedes tritt an die Stelle von Ūsingš und dem hl. Georg die poetische Vorstellung baltskarite (eine metaphorische Bezeichnung für Hafer) (30053,1-2). Das Bild aber wird Kunstvoll verzerrt, wenn auch dieser aufgefordert wird, entsprechend dem Grundtext, das 
Pferd zu striegeln und sich dann ans Trogende zu setzen! Mit anderen Worten, ein Text mythologischen Inhalts hat seinen mythologischen Gehalt verloren und ist zu reiner Dichtung geworden.

Der Einblick in die Varianten von Üsiņliedern, in denen wir den hl. Georg antreffen, zeigt ein sehr aussagestarkes Bild. Genau wie Ūsiņš, reitet auch hl. Georg zur nächtlichen Hütung, wie es eine Variante (30058) unseres Liedes 9 bezeugt. Die Texte sind identisch, einzig in der ersten Zeile ist hl. Georg an die Stelle von Üsinšs getreten. Auch hier zeigt eine größere Anzahl von Texten ein Abgleiten in reine Dichtung und so sind in Liedern sonst gleichen Inhalts auch andere die Reiter zur nächtlichen Hütung: die Schnepfe (30058,1; 30065,1; 51575-9), der Star (30069-30073; 51581) u. a. Überhaupt ist hl. Georg ein großer Reiter und das ganz besonders in der Georginacht, damit der Sommer allgemein gut wird:

Jurïts jāja Gurǵu nakti

Sirmu, bēru kumelinu,

Lai aug lava [?] vasarina, Kumeliniem barïbina. 51571
Jurits ritt in der Georginacht ein graues, braunes Pferd dim., daß ein guter Sommer dim. wachse, für die Pferde dim. Futter dim.

Das nächste Motiv, das hier auch zu erwähnen ist, ist der in dem 11. Lied erwähnte Ūsinšs der ,richtete' sich hoch auf“, und den man dann bittet, er möge doch in den Stall oder ins Haus hereinkommen und sich als Ehrengast ans Tischende setzen. Dasselbe wird von hl. Georg gesagt:

Jurḡīts stāvu slaistījāes

Mana stallla pakal̦ā.

Nãc, Jurginti, istabā,

Sēsties galda galiñā. 30059
Jurgits richtete sich hoch auf hinter einem Pferdestall. Komm, Jurgíiti, ins Zimmer, setz dich ans Tischende dim.

Die eben genannten Motive über den Reiter und den Pferdefütterer bieten keinerlei Schwierigkeiten bei der Identifizierung von Ūsinš̌ und hl. Georg und ihrem Vermögen nebeneinander aufzutreten. Das Bild wird sehr viel fremdartiger, wenn der hl. Georg sich hinter dem Stall hochrichtet. Da hilft die Erinnerung daran, daß in anderen Texten davon die Rede ist, daß „Üsinšs tanzte, Ūsiņš sprang" (12) hinter dem Stall. Dem Überlieferer der Vorstellungen war es schwieriger, diese mit dem christlichen Heiligen zu verknüpfen, deshalb fehlt es auch an derartigen Aussagen über ihn.

Das letzte Motiv, das über das Auftreten von Usiņš und hl. Georg nebeneinander, ist sehr bezeichnend, nämlich das Opfern. Bei der Erörterung unserer Texte haben wir bemerkt, welch zentrale Stellung das Opfern eines Hahnes im Üsiņš-Kult einnimmt (27-30). Auch dem hl. Georg wird ein Hahn geopfert ${ }^{1}$ :

1 Über die Opfer, die mit dem Austrieb des Viehs im Frühjahr zusammenhängen und die später auf hl. Georg bezogen wurden, besteht eine umfangreiche literatur (Cf. 
Jurīšam gaili kāvu

Deviniem nadziniem,

Lai tas manus zirgus gana

$\bar{s} \bar{a} d u$ garu vasarinu. $\quad 30057$
Dem Juritis schlachtete ich einen Hahn mit noun Krallen dim., damit or meine Pferde hüte diesen Iangen Som. mer.

Daß ein solches Opfer mit einem Opferversprechen verbunden war, zeigt aufs klarste der folgende bezeichnende Text:

To es došu Jurĝtĩăam,

Ko es biju solïjies:

Mucu alus, cepli maizes,

Melnu gaili k,ikaraini. 30075
Ich werde Jurgititis dim. das geben, was ich versprochen hatte: ein Faß Bier, einen Ofen Brot, einen schwarzen Hahn, den Kräher dim.

Daraus erhellt, daß der Text in Wahrheit von der Vorbereitung des uns schon bekannten kultischen Mahles spricht. Obwohl die Tradition des Opferns und des Mahles bezüglich des U̦sinš weit verbreitet war und in unserem Text gut dokumentiert ist, hat sie sich nicht in gleichem Maße mit hl. Georg verbunden. Tatsächlich sprechen davon mit klaren Worten nur diese wenigen Texte. Das scheint daran zu liegen, daß auch dieser Zug schwerer mit christlichem Bewußtsein zu verbinden ist.

Als wir von Ūsinšs sprachen, vermerkten wir, daß ihm ein Rock genäht bzw. geopfert wird (31); dem hl. Georg wird statt dessen ein Gürtel geopfert:

Jurğt̃̃̌am jostu aužu

Deviniem dzīpariem,

Lai tas manas aitas gana

$\$ a \bar{d}$ garu vasarinu. $\quad 30057,2$
Dem Jurgítis werde ich einen Gürtel weben mit noun Mustern, damit er meine Schafe hüte diesen langen Sommer dim.

Dieser Text, wie auch desselben Liedes $3 . \mathbb{V}$., zeigen, daß hl. Georg ebenso wie Usinš vereinzelt auch als Patron der Schafe und Kühe erscheint. Ein Überblick über die mit den beiden Gestalten - Ussiņš und hl. Georg zeigt, daß sie beide vorwiegend als Reiter bzw. Besitzer von Pferden miteinander identifiziert werden, oder als die, die für die Pferde sorgen. Andererseits ist festzustellen, daß mit hl. Georg verbundene Traditionen im Vergleich zu den Nachrichten über Ūsiṇš dürftig sind. Hier sei nur an die umfangreichen und wiederholten Beschreibungen von kultischen Mahlen und

HDA 3, 651 s), aus der ich nur einige Werke nenne. So Jahn 302: ,Wenn im Erzgebirge das Vieh zum ersten Mal auf die Weide getrieben wird, läßt der Hirt gekochte Eier, die er unter die Herde streut, vom Vieh zertreten und vergräbt die Schalen; so bleibt das Vieh beisammen. In der Mark Brandenburg wird das Vieh beim ersten Weidegang über ein Ei geführt. In Hinterpommern stößt man jodem Rind ein rohes Ei in das Maul, damit es fett und rund werde." Wir bemerken insbesondere, daß auch hier Eier eine besondere Bedeutung haben, wie das wiederholt auch in unseren Opfertexten der Fall ist. Cf. auch Haberland 59. 
anderen kultischen Handlungen erinnert. Man kann gewiß einwenden, daß Texte derartigen Inhalts über hl. Georg den aufschreibenden Personen weniger interessant waren und deshalb nicht intensiv genug gesammelt worden sind. Derartige Überlegungen ändern aber nichts an der jetzt vorliegenden Situation. Weiter fällt auf, daß in diesen Texten die Intimität fehlt, die wir bei Ūsiņš sehen, der als guter Mann, als alter Bruder, als Väterchen u. a. m. angeredet wird. Wodurch das begründet ist, ist schwer auszumachen. Das Auftreten von hl. Georg neben Ūsiņš in dessen Funktionen ist deutlich bezeugt, wiewohl dieses viel blasser ist. Bevor wir uns aber über dieser beiden genetischen Zusammenhang äußern, müssen wir uns einem Lied mit mehreren und dem Inhalt nach rätselhaften Vorstellungen zuwenden.

Besondere Beachtung fand in der Diskussion über Ūsiṇš das Lied 36:

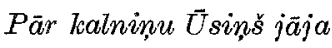

Ar alcmin' kumetinu.

Tas atnesa kokiem lapas

Zemei zalu äbolinu.
Über den Hügel dim. ritt $\overline{\text { Usiṇš }}$

Mit einem Rößlein aus Stein

Ex brachte den Bäumen Blätter

Der Erde grünen Klee. A 40

Auniņš hat diesen Text in $2 \mathrm{~V}$. mit sehr geringen Abweichungen veröffentlicht. Er hat ihn auch kommentiert. Zuerst weist er nochmals auf die bekannte Tatsache hin, daß man sich Ūsiņš ,,als einen gewaltigen Reiter vorgestellt habe. Dann versucht er zu erklären, wie das im Texte genannte Steinpferd und der Berg zu verstehen seien. Zum ersten hält er für möglich: ,Vielleicht geht der Ursprung des Liedes bis in jenes ferne Altertum zurück, wo die Letten weder Eisen noch Stahl kannten. "1 In Bezug auf den Berg bedürfe es keiner weiteren Erklärungen, um zu erkennen, daß darunter der Himmel zu verstehen sei². So richtig die letzte Einsicht ist, so falsch ist die Behauptung, unser Text sei in der Steinzeit entstanden, wie sich das bei der weiteren Analyse des Textes zeigt.

Schon 1873, acht Jahre vor Auniṇš, hatte Brōrzemnieks einen solchen Text veröffentlicht ${ }^{3}$ :

Tej atjāja brunots vīrs, Akmenotu kumelinu;

Tas atnesa kokiem lapas,

Zemätei zaļu zāli.
Hier kam herangeritten ein geharnischter Mann,

Auf einem Rößlein aus Stein:

Der brachte den Bäumen Blätter

Der Erde dim. grünes Gras. A

\footnotetext{
1 Auning $1881,31$.

2 Ib. ,Der Berg in den Liedern Nr. $[19,34,36]$ ist wohl nichts anderes als die Him. melshöhe, über welche die Sonne hinauf und herabsteigt und auf der sie zu ruhen scheint. Das Bild ist dem Volkslied und Märchen der Letten [...] sehr geläufig."

ง Brìvzemnieks 1873, 52, Nr. 190; auch Auniņš 1881, 32 und nachher 30063, 1 .
} 
Noch 5 Jahre früher, schon 1868, hat Sproǵis den folgenden Text veröffentlicht:

Kas tas bija, kur atjāja, Ar dūmainu kumelin??

Kas atnese kokiem lapas, Zemei zalu dāboltin?
Wer war es, der da herangeritten kam Mit einem dunkelgrauen Rößlein: Wer brachte den Bäumen Blätter, Der Erde den grünen Klee dim.?

Im ersten dieser beiden lieder ist vom gerüsteten Mann die Rede, im zweiten wird in Frageform geforscht, wer dieser Mann sei. Bei der Beurteilung dieser beiden mit dem anonymen Reiter nennt Auniņš seine Erklärung: „Im Sinne der alten Letten müßte auf die Frage des letzten liedes einfach mit 'Uhßing' geantwortet werden."2 Diese Frage aber ist weder so einfach, noch so sicher zu beantworten, wie Auniņs das getan hat.

Außer den beiden Texten von Auniņš selbst und je einem von Sprogiis und von Brīvzemnieks finden sich zwei diesbezügliche auch in den $\mathrm{LD}$ 30063 und 34067 - der erste mit 5, der zweite mit $16 \mathrm{~V}$. In jüngster Zeit wurden in $\mathrm{LTdz}$ zwei weitere Texte veröffentlicht $(1,2556-57)$. So ist die Gesamtzahl der Texte auf 29 angewachsen, 3 von ihnen sind je zweimal abgedruckt, so daß die wahre Anzahl 26 beträgt $^{3}$. Man kann sagen, daß alle diese Texte inhaltlich identisch sind. Unterschiede treten nur in den beiden ersten Zeilen auf. Die 3. Zeile stimmt fast wörtlich überein, einigemal tritt, was bedeutungslos ist, an die Stelle von atnesa 'trug herbei', atveda 'führte herbei' $(34067,6)$. Ein wenig zahlreicher sinn die Unterschiede in der 4. Zeile, wo an die Stelle des gewöhnlichen zalu äbolinu 'grünen Klee', das Synonym zalı zā̄li 'grünes Gras' tritt (34067,7-8), oder metaphorische Ausdrücke wie zal̦ villainīti 'grüne Decke'. Ein einziger Text nur $(34067,1)$ steht völlig außerhalb dieses Vorstellungskreises; auf den kommen wir später zurück.

Das Bild ist also einheitlich, die Texte stimmen in der formalen Gestaltung überein und sie beziehen sich auf die Ausschlagezeit des Laubes, also den Frühling. In den 26 Texten treten verschiedene Personen auf: in 5 Texten (36; 30063; 64067,6; LTdz 1,2557 wird Ūsinš genannt, in 6 Texten ,geharnischter Mann" (der Text von Brīvzemnieks; 30063,1), in 2 Texten Gott $\operatorname{dim} .(30063,1 ; 34067,6)$, in 13 Texten wird nach dem unbekannten Reiter gefragt und in einem ist von dem steinernen Mann die Rede (34067,1). Völlig aus dieser Reihe ausschließen können wir den Text 30063,2. Barons hat ihn offenbar nur als Variante an 30063 angeschlossen, weil er in der 3.

1 Sprogíis 300; auch 34067,6 .

2 Auniņš 1881, 32.

${ }^{3}$ So entspricht 30063, 1 dem Text von Brivzemnieks under 34067,6 dem von Sproǵis. 
und 4. Zeile völlig mit den anderen Texten dieser Gruppe überinstimmt. Die erste Hälfte mit der 1. und 2. Zeile aber führt uns in eine völlig andere Welt:

Es satiku vecu viru

Vecumāi staigājot.

Ich traf einen alten Mann, wandernd über altes Land.

Mit vecumāi Loc. sgl. 'im alten Land' ist Brachfeld gemeint. Wer der alte Mann ist, und warum er über das Brachfeld wandert, gibt der Text nicht an. Die Vorstellung vom alten Mann ist unter den Wesen, die die Fruchtbarkeit der Äcker fördern, fremd. Am glaubhaftesten ist die Annahme, daß der Text unter dem Einfluß der anderen Texte dieser Liedergruppe entstanden ist, ohne direkt zu ihr zu gehören'. Jedenfalls paßt die in der ersten Hälfte des Textes genannte Person nicht zu den anderen in dieser Liedergruppe genannten Personen. Ebenso fremd ist akmentiña vīriņš 'ein steinernes Männerchen' $(34067,1)$, aber darüber wird später zu reden sein.

Mit dieser Liedergruppe hat sich vor allem Kreics beschäftigt und den Versuch unternommen zu erklären, wie sie überhaupt zu verstehen ist, und welche Person oder welches Wesen ursprünglich in diesen Texten aufgetreten ist, in denen jetzt Wesen mit verschiedenen Namen genannt werden. Er argumentiert folgendermaßen. Kreics verfaßte seine Schrift in scharfer Polemik gegen Brastinss. Dieser hatte sich auf 30063 berufen²:

$P a$ zāläti Dieviñš brauca Akmenoju kumelinu, Tas atveda kokiem lapas, Zemei zaḷ u äbolinu.
Übers Gras fuhr Dievs dim. auf einem Rößlein aus Stein, der brachte den Bäumen Blätter, der Erde grünen Klee.

Ähnlich auch 34067,6-2 V.

1 Es besteht gar kein Grund dafür, diesen Text so zu verstehen, wie es Kreies, 217, wünscht: „In der Variante taber den alten Mann ist, scheint mir, nicht von hl. Georg, sondern von einem alten Priester die Rede, der am Georgstag beim Bittgang über den Acker geht, ihn segnet und mit heiligem Wasser besprengt. Der Autor dieser Variante beschreibt zweifelsohne ein von ihm selbst beobachtetes Ereignis - den Bittgang, den in alten Zeiten der alte Priester durchführte." Wir werden später sehen, daß diese Behauptung des Kreics in dessen eigener Überzeugung wurzelt. Jetzt aber schon kann man anmerken, daß die Behauptungen über den alten Priester, über das Versprengen von Wasser, über den Georgstag u. s. w. im Text keineswegs dokumentiert sind. Noch weniger verständlich ist, welchen Grund der Autor dafür hat, zu behaupten, der Text berichte das von einem Augenzeugen Gesehene. Hier genügt es, daran zu erinnern, daß man ebensogut, wie es Frazer unter Berufung auf Mannhardt tut, meinen kann: ,Commonly the spirit of the ripe corn is conceived, not as dead, but as old and hence it goes by the name of the OLD Man or Old Woman"s (Frazer 3, 253 s). Insoferm entbehren die Vermutungen des Kreics jeder Bedeutung; sie finden keine Begründung im Material.

2 Cf. Brastin̨š 1928, 19. 
Brastiņš meint, es handele sich um einen altlettischen Gott Dievs, der den Bäumen Blätter und der Erde grünen Klee bringt. Kreics polemisiert dagegen und behauptet, Dievs sei später in den Text eingedrungen'. Wie lauten nun seine Argumente? Erstens beruft er sich darauf, daß das Wort Dievs nur in $3 \mathrm{~V}$. dieser Tiedergruppe auftritt, die dazu noch in zwei Nachbargemeinden aufgezeichnet wurden. Zweitens erkennt er im Liederinhalt Widersprüche. Die übrigen Texte sprechen vom Reiter, der den Bäumen Blätter bringt, Dievs aber ist ein Fahrer. Dievs fährt auch, ,übers Gras“", in der zweiten Liederhälfte aber heißt es, er bringe Blätter und Klee. Das erscheint dem Kreics ein unmöglicher Widerspruch: wenn Dievs übers Gras fahren kann, muß es doch auch schon Blätter geben. Drittens weichen diese Texte formal von der Frageform ab, in der in den anderen nach dem anonymen Reiter gefragt wird. Hier ist die Frage schon beantwortet und es ist gesagt, da $\beta$ es Dievs sei. Weiter wendet er ein, daß, wenn in der zweiten Hälfte der Texte die Erklärung für das Bringen der Blätter ,beibehalten wird, darin das persönliche Führwort viņš 'er' und nicht das demonstrative Führwort tas 'der' zu erwarten wäre".

Mit anderen Worten, Kreics sieht die Texte mit der Frageform und dem anonymen Reiter als die älteren an. Er macht dem Herausgeber der LD Barons überhaupt den Vorwurf, fälschlicherweise Lieder in Frageform in seine Sammlung aufgenommen zu haben, denn das seien in Wahrheit Rätsel. Zu diesen Gedanken von Kreics ist zu bemerken, daß das erste Argument, daß nämlich nur 3 (in Wahrheit 2) Texte für Brastiņš sprechen, die noch dazu in geographisch sehr engem Raum aufgeschrieben wurden, zu beachten ist. Aber das numerische und das geographische Argument reicht an und für sich nicht aus, um die Tatsache, daß zu diesen Texten ursprünglich Dievs gehört, zu bezweifeln oder zu beweisen. Thm erscheint es auch als Widerspruch, daß Dievs, der übers Gras fährt, nun erst die Blätter bringt. In Wahrheit ist das kein Widerspruch, denn in der Natur wird das Gras ja längst grün, ehe die Bäume ausschlagen. Wenn aber Kreics nun durchaus einen Widerspruch darin erkennen will, kann man

1 Kreics 213: „Diese Beobachtungen beweisen, daß die Varianten mit Dieviñ um in heutiger Sprache zu sprechen - Plagiate sind, und zwar sehr ungeschickt verfaßte; von der alten, ersten Fassung des Liedes sind nur die 2 letzten Zeilen übriggeblieben, die beiden ersten aber sind später angefügt, vielleicht einige Jahrhunderte nach der Entstehung der ersten Fassung, zu einer Zeit, da der erste, echte Reiter schon vergessen war, und das Lied in seiner ursprünglichen Fassung der Generation der Plagiatoren unverständlich geworden war. " Die Behauptung über Plagiate zeigt, daß Kreics den Vorgang der Entstehung und der Tradierung von Volksüberlieferung nicht verstanden hat. Volksüberlieferung befindet sich inmer im Prozess der Dynamik and nimmt daher stets neue Elemente auf. Das aber ist kein Grund, von Plagiat zu sprechen. Kreics hat viel behauptet; die Beweise fehlen. 
darauf verweisen, daß 'einen solchen zwar der systematisch denkende Mensch erkennt, die Volkskunst aber weder vom systematischen Denken bestimmt, noch dessen Ergebnis ist. Ganz allgemein bedeutet Dichtung Abstand vom Realen. Ebensowenig hat die von Kreics in diesem Zusammenhang gemachte Zusatzbemerkung Bedeutung, nach der die 3. Zeile mit dem persönlichen Führwort viņš und nicht mit dem demonstrativen Führwort tas hätte beginnen müssen. Dieses grammatische Gesetz ist eines der schwächsten in der praktischen lettischen Sprache, das sogar von hochgebildeten Sprechern des Lettischen übertreten wird ${ }^{1}$. Daß das auch in der Volksdichtung so geschieht, versteht sich von selbst.

Ohne Bedeutung für die Erörterung des Inhalts ist das Argument von Kreics, ein größerer Teil der Lieder hätte Frageform, oder sie seien, wie er sagt, Rätsel. Die Lieder mit Dieviņš seien in Frageform verfaßt und hätten deswegen sekundäre Bedeutung. Er irrt auch bei der Annahme, diese Lieder seien beiseite zu lassen ${ }^{2}$. Auch wenn sie Rätsel wären, ändert das nichts an dem von uns zu erörternden Inhalt. Die Frage lautet doch, wer der anonyme Reiter ist. Es ist immer derselbe, unabhängig davon, in welchem Folkloregattung und in welcher Sprachform die Quelle verfaßt ist. Es ist ein Irrtum, wenn Kreics die in Frageform verfaßten Texte als Rätsel ansieht. Tatsächlich sind rhetorische Fragen in den lettischen Dainas eine sehr verbreitete Erscheinung ${ }^{3}$. Diese Einwände erweisen die Argumente von Kreics als nicht so überzeugend, daß man allein auf ihnen fußend anerkennen müßte, der in diesen Texten genannte Dievs sei später eingedrungen. Wie wir später sehen werden, ist das Problem viel komplizierter. Weiter erwähnt Kreics, daß in diesen Liedern auch Ūsiņ̌s als Reiter genannt wird. Darüber aber gleitet er leicht hinweg. Er konstatiert: ,Allein auf Grund der lettischen Volkslieder, in denen so viele und unterschiedliche Bringer von Gras, Klee und Blätter genannt sind, diese Rätsel zu lösen, ist unmöglich. Dennoch

I Davon kann man sich auch in der Schrift von Kreics selbst überzeugen, denn auch er unterscheidet Personal- und Demonstrativpronomina nicht konsequent, z. B. ,Der in ihm [dem Lied] genannte Reiter ist dem [Barons] auch nur noch zu etwas Geistigem, U̇bernatürlichen geworden" (Kreies 212). Schon Auniṇš hatte Šmits gegenüber zu seiner Zeit wohlbegründet darauf hingewiesen, daß sprachliche Fehler eher auf die Echtheit als auf eine Fälschung der Volksüberlieferungen schließen lassen (cf. Auniňs 1913, 26).

2 Kreics wirft Barons vor: , Diese Form beweist, daß dieses Lied kein Lied im wahren und echten Sinme des Wortes ist, sondern ein Rätsel, dessen Lösung der Name des im Text genannten Reiters ist. Kr. Barons hat das nicht erkannt, hat dieses Lied nicht als Rätsel aufgefaßt und nicht versucht, seine Lösung zu finden" (Kreics 212). Der Vorwurf ist ungerechtfertigt; Barons hat sich nicht mit der Erforschung der lettischen Mythologie befaßt, sondern nur die ihm eingesandten Lieder gruppiert.

3 Cf. Biezais 1961, 163. 
weisen die Varianten mit dem bewaffneten Mann den Lösungsweg. "1 Hier ist nun an das erstmalig von Brīvzemnieks 1873 veröffentlichte Lied mit dem bewaffneten Mann zu erinnern. Neben diesem sind, wie gesagt, noch 5 ähnliche bekannt $(30063,1 ; 34067,2-3 ; 34067,7-20)$, von denen wir hier eins abdrucken:

Atjäj viens brunots vīrs
Par lielo tīruminu
Tas atnesa kokiem lapas,
Zemei zal̆u äbolin, $\quad \mathbf{3 4 0 6 7 , 2}$

\begin{abstract}
Angeritten kommt ein bewafineter Mann über das große Feld dim.; der brachte den Bäumen Blätter, der Erde grünen Klee.
\end{abstract}

Nach seiner Meinung ermöglicht das Material keine Antwort auf die Frage, wer der bewaffnete Mann sei, und deshalb verweist er auf Material, das bei den Weißrussen zu finden ist. Das ist verständlich, denn die Weißrussen sind die nächsten Nachbarn der Letten im Osten. Er erwähnt 3 derartige Texte:

Святол Ягорья - Бозкий ключник, Уаяу клюии - атамкнуу зямлю, Атамкнуу зямлю - пусьциу расу. ${ }^{2}$

A 1 Opьrea namb

$\Pi$ I $а$ eоpe хадила,

Кллючики насила,

3лялю атмыкала,

траву выпускала,

На травичу расиџу.

IOрава мачі упроч паила, Залатья ключь панясла, Юорева мачі вярнісл:

Залатыл кллочь наиліся.

Адамкні зямлічу,

Bünyсbyi paciyy,

На зялену травіцу,

Ha здраеву вадзічу. ${ }^{3}$
Der hl. Georg - der göttliche Schlüsselträger, nahm die Schlüssel - schloß die Erde auf, schloß die Erde auf ließ Tau fallen.

Die Mutter Georgs aber wanderte über den Berg, sie trug die Schlüssel dim., [sie] schloß die Erde auf, [ließ] das Gras sprießen, [tränkte] das Gras mit Tau.

Die Mutter Georgs ist fortgegangen, hat die goldenen Schlüssel weggetragen. Mutter Georgs, komm zurück: die goldenen Schlüssel haben sichn eingefunden. Schließ die Erde dim. auf, tränke mit Tau dim. das grüne Gras dim., das Wasser dim. der Quellen.

Ohne eine genauere Analyse dieser weißrussischen Texte, oder deren kritische Überprüfung sagt Kreics summarisch: „,Diese weißrussischen Texte stimmen zwar nicht Wort für Wort mit den Texten unserer Lieder überein, aber ihr Grundgedanke ist derselbe: Der Georgstag und dessen Nacht schließt die Erde auf, bringt Gras, Tau, den Bäumen Blätter. Das heißt, mit

1 Kreics 214.

2 Romanov 174; 183. Dieser und der nächste Text ist aus Kreies, $214 \mathrm{~s}$, übernommen.

a Časopis 29. Dieser Text ist aus Kreics, 215, übernommen. 
dem Georgstag beginnt der Graswuchs, das Ausschlagen der Blätter. Derselbe Georg ist auch die Lösung der von uns zu betrachtenden Rätsel. " ${ }^{\text {1 }}$

Seine Auffassung versucht er weiter damit zu begründen, daß der hl. Georg bei den Slaven sehr geliebt gewesen sei. ,Von den Russen übernahmen die Letten mit dem Christentum auch den Georgskult vor dem Eintreffen der Deutschen, und der Name Georg war auch bei den Letten vom Beginn der Christianisierung so verbreitet, wie bei den Russen."2 Er begründet seine Behauptung mit der Verbreitung dieser Lieder gerade in den Ostgebieten Lettlands. Er hat diese Lieder auch datiert: „, Deshalb ist anzunehmen, daß die Lieder über den bewaffneten Reiter im Bereich von Krustpils, Sece, Sēḷpils und Livāni in ihrer ursprünglichen Redaktion in diesen zwei Jhen., dem 11. und 12., entstanden sein werden. In derselben Zeit mögen auch die Varianten aus Bērzaune und Vestiena entstanden sein. Damals gehörten diese Gebiete rechtgläubigen russischen Fürsten. Nicht früher, als im 11. Jh., wo die Letten noch nicht getauft waren, die Heiligenbilder Georgs noch nicht gesehen und die Feier des Georgstages noch nicht erlebt hatten und nicht später, als im 12. Jh., wo die Letten schon unter deutscher Herrschaft standen, und Gebete zum hl. Georg vor dessen Bilde am Georgstage nicht mehr stattfanden, mußten diese Lieder bzw. Rätsel entstanden sein. Deshalb wurden sie später mannigfach variiert, verändert. Es ist anzunehmen, daß mit den ersten Anbetungen auch die ersten Lieder entstanden."3

Zur Argumentation von Kreics ist folgendes zu bemerken. Sein Hinweis auf das Eindringen des hl. Georgs in unsere Liedergruppe ist richtig. Das bestätigen eindrucksvoll, wie wir in unserer Erörterung gesehen haben, die lexikalischen und die Folklorematerialien. Die Berufung auf das weißrussiche Material erbringt nichts Neues. Das wäre nur dann der Fall, wenn aus diesem Material hervorginge, welche Funktionen der hl. Georg von einem vermutlichen vorchristlichen Wesen dort übernommen hätte. Daß der Kult des hl. Georgs wie in der Ostkirche, so auch in der Westkirche weit verbreitet war, ist allgemein bekannt ${ }^{4}$. Das hat zu verschiedenen Erschei-

1 Kreics 215.

2 Ib. 216.

3 Ib. 218.

- Wie kompliziert und widersprüchlich die Gestaltung des Georgsbildes unter dem Einfluß der anderen orientalischen Religionen im Christentum gewesen ist, darauf haben mehrere Forseher hingewiesen. So hat Clermont-Ganneau, wiewohl er in der Suche nach Beziehungen zu weit gegangen ist, in seiner Studie am Ende des vorigen Jahrhunderts, die Vereinigung der fremden Elemente selbst im Prinzip richtig gesehen: ${ }_{,}$Ce qu'il y a de plus surprenant c'est que ces deux états de la légende semblent être représentés par une même individualité, par ce Khidhr énigmatique, qui équivaut d'une part à saint Georges, Horus, Persée, et de l'autre s'affilie au groupe typhonien et mari- 
nungen des Synkretismus geführt, nicht nur im Christentum, sondern auch im Islam, so unter anderem im Fruchtbarkeitskult ${ }^{1}$. Die 3 von Kreics aufgeführten Texte ergeben eine recht schwache Bestätigung dieser bekannten Wahrheit, insofern als in ihnen die Aufschließerin der Erde und die Bewahrerin der Schlüssel die Mutter des hl. Georgs ist. In der christlichen Hagiographie ist sie unbekannt, und man könnte unter ihr eher die vorchristliche Mutter Erde vermuten ${ }^{2}$. Das ist aber eine der Forschung bedürftige Frage für sich.

Das Verdienst des Kreics besteht darin, daß er als erster den bewaffneten Mann mit dem hl. Georg identifiziert hat. In dieser Hinsicht ist seine Argumentation überzengend. Daß der hl. Georg in das lettische Material eingedrungen ist, entspricht auch vollkommen dem Gesamtbild. AuBer auf die von Kreics genannten Texte kann man noch auf einige weitere zu dieser Gruppe gehörende verweisen. Einer von diesen sagt, der unbekannte Reiter sei ,auf einem stählernen Pferd angeritten gekommen“ (34067). Das schließt sich direkt an den bewaffneten Mann bzw. den bewaffneten Reiter an. Fin zweiter Text, der in diesem Zusammenhang genannt werden kann, sagt, er sei ,auf einem silbrigen Pferdchen geritten“ (34067,1). An die Stelle des

time. Ces deux conceptions radicalement opposées sont venues se confondre sous un même nom (on a déjà comparé Гopүú et Té́pyos); j'essayerai de faire comprendre tout à l'heure qu'il y a bien autre chose encore dans Khidhr, et qu'à côté de ce dualisme contradictoire résumé en lui, il reste à en dégager d'autres éléments encore plus hétérogènes. J'aurai pour cela à interroger fréquemment la tradition arabe, dont on aurait grand tort de faire systématiquement fi, car bien so uvent elle parle, - mal, je veux bien, - mais enfin elle parle là où les autres sont muettes" (Clermont-Ganneau 389). Noch bestimmter hat sich Cumont, $40 \mathrm{~s}$, über die Georgslegende geäußert: ,Nous trouvons ainsi dans l'élucubration de cet hagiographe une preuve nouvelle de l'influence qu'ont exercée ces cuvres judéo-mazdéennes qui naquirent dans les pays, comme la Mésopotamie et l'Asie Mineure, où les rabbins et les mages se coudoyaient. Dans le syncrétisme de l'époque hellénistique, une certaine combinaison de leurs doctrines était aussi inévitable que le fut à Alexandrie l'action de la pensée grecque sur un judaïsme devenu philosophique. Parmi les apocryphes chrétiens qui recueillirent ce mélange d'idées perses et juives la légende de Georges, qui jouit d'une popularité immense, occupe de ce fait une place considérable. La fantasmagorie qui la caractérise est le fruit de sa double origine orientale." (cf. auch Küppers 5). Krause 1893, 205 ss, hat versucht, die Gedanken von Clermont-Ganneau, freilich weitgehend unkritisch, auch auf die nordischen Völker zu beziehen.

1 Das zeigt schon das griechische Wort selbst. So Clermont-Ganneau 388: „Le nom

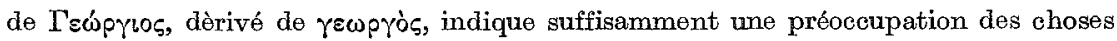
agricoles; le saint subit, entre autres, un supplice que rappelle la meule. Le nom arabe de saint Georges, Khidhr, veut dire vert, verdoyant, parce que partout où Khidhr mettait le pied ou s'asseyait, la terre se couvrait de verdure." Über die Identifizierung von hl. Georg und Khidr ef. auch Biezais 1976.

2 Über das Problem der Mütter in der Religion der Letten Biezais 1955, 258 ss, 286 ss. 
in Metall gekleideten Mannes tritt in diesen Texten freilich das metallene Pferd. Die Behauptung von Kreies, der Kult des hl. Georgs bei den Letten sei von den Russen übernommen, ist nicht zu beweisen. Daß er ,auch bei den Letten von Beginn der Christianisierung so verbreitet war, wie bei den Russen", ist eine bloße Behauptung, für die Kreics keinerlei Begründung gibt"1. Eine Begründung läßt sich auch nicht finden, weil hierüber Nachrichten in den Quellen fehlen. Hier muß auch daran erinnert werden, daß bloß eins der von Kreics genannten Lieder im östlichen Lettland, unweit der russischen Grenze aufgeschrieben worden ist. Dagegen läßt sich gewiß einwenden, daß von dort her vielleicht überhaupt weniger Lieder eingesandt worden sind. Der Hinweis von Kreics, die Lieder seien unter dem Einfluß des Protestantismus geschwunden, ist kaum glaubhaft, denn die lettische Ostprovinz ist nie protestantisch gewesen, sondern hat ihren katholischen Charakter bewahrt.

Gegenüber der Behauptung, die Rolle des hl. Georgs bei den Letten sei von den Russen entlehnt, kann man ebensogut das Gegenteil behaupten, nämlich den Einfluß der Deutschen. Schon seine Bezeichnung als brunots vīrs 'bewaffneter Mann', spricht eine verräterische Sprache, denn brunots ist ein Germanismus ${ }^{2}$, und kann demnach nur unter dem direkten Einfluß des Deutschen entstanden sein.

Kreics kann auch keine besonderen Kultstätten in Ostlettland nennen, wo der Georgskult gepflegt worden wäre. Dagegen war der hl. Georg ein sehr wichtiger Mitpatron des das Land erobernden Deutschen Ordens ${ }^{3}$.

1 Über die Verbreitung und den Kult des hl. Georgs bei den Russen sind zwei gute Arbeiten zugänglich: Veselovskij, besonders $156 \mathrm{ss,} \mathrm{und} \mathrm{Kirpičnikov} 228 \mathrm{ss} . \mathrm{Zu}$ den Arbeiten Veselovskijs und Kirpiǒnikovs vgl. Heinzel 257 ss. Über die Südslaven hat sich insbesondere Krause, 1893, 213 ss, geäußert. Zu seinen Einsichten hat er bemerkt: „Was nun hier vorzugsweise von slavischen Völkerschaften berichtet wurde, darf ziemlich unbedenklich auf die germanischen übertragen werden; denn so verschieden auch ihre sonstigen religiösen Vorstellungen gewesen sein mögen, bemerken wir doch gerade in der Feier der Sonnenlauffeste (Weihnachten, Ostern, Johannis und Michaelis) die größte Übereinstimmung. In der teilweisen Verlegung unserer Ostergebräuche auf das hl. Georgsfest können wir sogar unschwer ein Festhalten an älteren vorchristlichen Vorstellungen erkennen." Hier ist besonders seine richtige Erkenntnis zu unterstreichen, daß solche Züge vielen europäischen Völkern gemeinsam sind.

2 Zēvers 385: „Für den Harnisch haben die Letten keinen einheimischen Namen, sondern ein Lehnwort brunas, dessen erste Quelle im Germanischen zu suchen ist, vgl. got. brunjo und ahd. brunnia. Aus dem Germanischen stammt asl. branja 'Panzer', das wahrscheinlich weiter als brunas ins Lettische entlehnt ist." Da das Wort schon in den ältesten lettischen Sprachdenkmälern belegt ist, die von Deutschen verfaßt sind, braucht man nicht von einem slavischen Einfluß zu sprechen.

${ }^{8}$ So Hein 387: ,,Das Kompatrozinium dieses Heiligen über den Deutschorden ist darauf zurückzuführen, daß Georg im Kampfe der Ordensritter gegen die Sarazenen den ersteren als mächtiger Helfer erschienen ist und die Brüder zum glänzenden Siege 
Ihm waren auch besonders im Ordensterritorium mehrere Kirchen und Kapellen geweiht ${ }^{1}$. Das bedeutet daß ein solcher Einfluß durchaus auch in der Zeit der Wirksamkeit der Deutschen bzw. der katholischen Kirche entstanden sein kann.

Auch ikonografische Motive lassen solche Gedanken zu. Kreics beruft sich darauf daß die Kiever Russen um 989 getauft worden seien daß das Bistum Polozk um 1000 gegründet worden sei und fährt dann fort: ,Deshalb ist anzunehmen, daß um diese Zeit, also zu Beginn des 11. Jhs., jedenfalls aber in dessen erster Hälfte, die Christianisierung der den Fürsten von Polozk unterstellten Letten oder Lettgaller begann und bis zur Ankunft der Deutschen fortgeführt wurde. ${ }^{62}$ Diese wird um 1186 datiert. Da ist zuerst zu bemerken, daß von einer ernsthafteren Kenntnis des christlichen Glaubens, geschweige denn von einer Verwurzelung in demselben in der Zeit von 1000-1186 nicht die Rede sein kann ${ }^{3}$. Die Unterwerfung unter die Fürsten von Polozk war ein lockeres Tributverhältnis. Auch die neugetauften Russen lebten weiterhin tief verwurzelt in vorchristlichen Traditionen. Die byzantinische Kunst, unter deren Einfluß sich Vorstellungen vom hl. Georg bei den Russen bildeten, kennt ihn bis ins 12. Jh. hinein als, jugendlich, bartlos in byzantinischer Hoftracht in langem Rock, mit Mantel darüber dargestelltt ${ }^{6_{4}}$. Wenn er als Kämpfer auftritt, dann ,zu Fuß mit dem Panzer gekleidet und mit Lanze und Schild“. Denkmäler mit dem hl. Georg zu Pferde als Drachentöter, ,kommen erst vereinzelt in der östlichen Kunst seit dem 12. Jh. vor, und sie verdrängen auch in der Folgezeit hier die übrigen Darstellungsformen nicht in dem Maße, wie in der Kunst des Abendlandes ${ }^{* 5}$. Künstle sagt bei der Behandlung der Firscheinung des $h l$. Georgs in der westeuropäischen Kunst, dieser sei bis ins 12. Jh. nach byzantinischer Tradition als der Friedensbringer dargestellt worden, erst in der ersten Hälfte des 13. Jhs. wird er als Drachentöter bekannt, aber immer noch jünglingshaft und zu FuB. Der Drachentöter zu Pferde ist eine späte ikonographische Erscheinung6. Die hier genannten historischen und ikonoführte." Cf. auch Bruiningk 413 ss. Über das Patrozinium des hl. Georgs besonders 420: "So war dieses Patrozinium in erster Linie dem zum Schutze der Christen in Livland gestifteten Ritterorden besonders nahe gelegt." Hier ist besonders daran zu erinnern, daB hl. Georg in der christlichen Ikonographie wiederholt im Rittergewand erscheint. Cf. Küppers, besonders 52, 56.

1 Hein, $390 \mathrm{~s}$, nennt mehrere dem hl. Georg gewidmete Kirchen und Kapellen. Nachrichten darüber auch bei Bruiningk 414; Kurtz, Edith, 92, 100 u. a.

2 Kreics 217.

s Darüber hat sich mehrfach auch der Kirchenhistoriker Adamoviěs geäußert.

4 Künstle 2, 266, 268; ähnlich auch Lexikon 6, 367; cf. auch Aufhauser 239.

5 Ib. Cf. auch Lexikon 6, 368.

6 Ib. 269. Lexikon 6, 378: „Drachenkampf zu Pferd (v, Adel bevorzugt). Dem byz. Typus bringen das 13. und 14. Jh. neue Züge zu. Kampf m. eingelegter Lanze beginnt 
graphischen Motive zwingen zu Zweifeln an die Hypothese des Kreics über den russischen Einfluß, insbesondere da er keinerlei konkretes Material zur Stützung seiner Ansicht beizubringen vermag. Zum mindesten kann man mit gleicher, wenn nicht gar mit größerer Sicherheit über den Einfluß der westlichen christlichen Kirche und des deutschen Rittertums sprechen.

Im Rahmen dieser Arbeit ist es nicht möglich, den Einflüssen im Einzelnen nachzugehen, die das Bild des hl. Georgs selbst in der christlichen Kirche gestaltet haben. Wir müssen uns also hier mit einigen Hinweisen begnügen. Der Reiter als Drachentöter, der in der christlichen Kirche so weit verbreitet ist, hat offenbar sehr ferne und alte Beziehungen. Vorbilder für ihn sind schon in vorchristlicher Zeit und vor allem beim Stiertöter des Mithras-Kults zu suchen ${ }^{1}$. „In Legenden östlicher Länder erscheint der hl. Georg in Verbindung mit einem Ochsen."2 Diese Symbolik spricht ihre klare und unmißverständliche Sprache. Das Bild des siegreichen Reiters kommt tatsächlich aus dem Iran ${ }^{3}$. Jedenfalls ist die Symbolik des MithrasKults nicht die einzige, die den Weg dahin weist, wie derartige Züge sich mit dem hl. Georg verbinden konnten. Dumont meint, auch andere antike

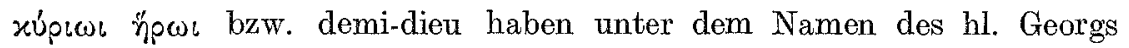
weitergelebt ${ }^{4}$. Man kann rein spekulativ auch annehmen, daß der Kampf

seit 13. Jh. bes. i. 14. Jh. d. ant. Art d. Lanzenstichs m. ausgedrecktem Arm (die weiter bis 16. Jh. dargest. wird) zurückzudrängen. Kampf mit Schwert vereinzelt seit 12. Jh., allg. seit 15. Jh" Aufhauser 237: ,Die ältesten uns bekannten Wunderberichte enthalten den Drachenkampf nicht. Die Legende des Drachenkampfes erscheint erst seit dem 12. bis 13. Jahrh. in der literarischen and künstlerischen Überlieferung."

1 Cf. Strzygowski 49 ss; Lexikon 6, 369; Siecke 111; mit reichem Bildmaterial and Bibliographie Hulst, Cornelia, 135-156, sowie auch mit Hinweisen auf persische und ägyptische Beziehungen. Cf. auf Kirpičnikov 46 ss, 59 ss.

2 Reinbot LXXVII. Ib.: ,[...] der Zusatz vom Drachenkampf ist erst unter dem Finfluß der symbolischen Darstellungen von Georgs Kampf mit dem Heidentum entstanden."

3 Strzygowski $58 \mathrm{~s}$, ,Der Weg aber [...] ist der vom Orient über die hellenistischen Großstädte, vor allem Alexandreia, und über das ionische Marseille an den Rhein, wo wir die Hauptbeispiele der Darsteliung des römischen Kaisers zu Pferd als des Siegers über die Barbaren finden. Der hl. Georg mag die letzte Erinnerung dieser Entwicklung sein oder nicht, jedenfalls hält er noch den altorientalischen Typus des Sieges des Guten über das Böse fest. Für den Ursprung dieses Typus weisen alle Wege in dieselbe Richtung, aus der das Pferd selbst vom Iran aus nach dem Westen vorgedrungen ist." Cf. auch HDA 3, 1333.

4 Dumont 219: ,Ce héros continue d'être adoré sous le nom de saint Georges. Sa ressemblance avec ce personnage chrétien, telle que la peinture byzantine, aujourd'hui invariable, le représente, est évidente. Dans l'église arménienne de Philippopolis, il a une place d'honneur, où l'on brûle des cierges comme devant une image orthodoxe. Dans nombre d'églises, le cavalier thrace est sealement sanctifié par une croix qui laisse subsister la dédicace païenne. A Tiristasis, il a fait donner le nom de Saint-Georges à une chapelle bâtie au milieu de ruines antiques, où on a trouvé un marbre sur lequel

$10-762518$ Biezais 
des hl. Georgs ein Urphänomen sei, dem der in der menschlichen Existenz wurzelnde Kampf des Guten gegen das Böse zugrunde liegt ${ }^{1}$.

Letzten Endes ist die Diskussion darüber, ob der ,bewaffnete Mann“ in den Dainas als metaphorische Bezeichnung für den hl. Georg unter russischem oder unter deutschem Einfluß entstanden ist, im Rahmen unserer Erörterung ohne große Bedeutung.

Jedenfalls ist die Gestalt des hl. Georgs und seine Bezeichnung als ,, bewaffneter Mann" bei den Letten unter christlichem Einfluß entstanden. Die Diskussion hat dennoch insofem Bedeutung, als nach Feststellung der Identität des ,,bewaffneten Mannes “ und des hl. Georgs, wir die 6 Lieder mit dem bewaffneten Mann zu den Liedern um den hl. Georg zählen können. Dennoch ist die Frage um das Auftreten von Usiņš — hl. Georg (bewaffneter Mann) - Dieviņš in dieser Liedergruppe immer noch nicht beantwortet. Noch weniger ist damit die Frage beantwortet, wer Usinš ist? $\mathrm{Zu}$ ihr kehren wir unter Berücksichtigung gerade dieser Liedergruppe nochmals zurück. Vorher aber ist den Frage nachzugehen, ob die Identifizierung des Usiṇš ausschließlich mit St. Georg oder auch mit anderen Heiligen der christlichen Kirche erfolgt ist.

4.2.3.2. Eine ähnliche Identifizierung erscheint auch zwischen Ūsiņš und dem Mārtiņš 'hl. Martin'. Viele Dainas sind völlig gleichen Inhalts und stimmen sogar wörtlich überein. Nur der Name Usiņš ist durch den Namen Mārtiņš ersetzt. Hier nennen wir nur ein paar uns schon sonst bekannter Texte. Mārtin̄š ist der Fütterer der Pferde.

Ei, Märtin, labais vīrs,

Baro manus kumelinus,

Sukādams, barodams,

Sēsties siles galiñä. $\quad 30209$
Ei, Martin, guter Mann, füttere meine Rosse dim., indem du [sie] striegelst, fütterst, setze dich an das Ende dim. des Futtertrogs.

Dies entspricht unserem 46, aber mit der intimen Anrede 'guter Mann',

est figuré ce demi-dieu national. II serait intéressant, pour l'histoire de l'art, de savoir quelle a été l'influence de cette représentation sur le type consacré de saint Georges, qui paraît avoir été fixé dès les premiers siècles du christianisme. La ressemblance est frappante, et je ne crois pas que le cavalier béotien, qui, du reste, attaque rarement une bête sauvage, ait servi de modèle aux premiers peintres chrétiens plutôt que le cavalier thrace."

1 Diesen Gedanken hat besonders Aufhauser, 245 ss, entwickelt: „All die verschiedenartigen Darstellungen eines Drachenkampfes bei den verschiedensten Völkern und Religionen verkörpern eben den einen allgemein menschlichen Grundgedanken: Kampf und Sieg des Guten über das Böse. Die Urform dieser Idee reicht zurück bis in die Urzeit der Menschheit selbst. So alt das Menschengeschlecht, so alt ist eben auch dieser Kampf. Orientalische Art aber ist es, den Sieg des guten Prinzips über das böse unter dem Bilde eines Kampfes mit einem Untier darzustellen. Und diese Einkleidung der Idee hat sich von der Wiege der Menschheit an die ganze Welt erobert." Cf. auch Krefting 12 ss, 34 s, 86; Strzygowski 59. 
die im Zusammenhang mit hl. Georg nicht vorkommt. Vgl. auch 30210. Genau wie Ūsiņš „,trieb sich der hl. Martin aufrecht hinter meinen Stall herum" (30209,1; 30226) und wie jener wird er aufgefordert, in den Stall zu gehen und die Pferde zu füttern. Es gibt aber keine Texte, die angeben, daß auch hl. Martin wie „Ūsiņš tanzte, Ū $\operatorname{sins}_{3} \operatorname{sprang}^{4}(12)^{1}$.

Der hl. Martin ist ebenso reich an Pferden (30214) wie Ūsinšs, deshalb kann man sich von ihm, wie von Ūsinš̌ ein Pferd erbitten:

Märtiņam pilns stallitts

Sirmu, bēru kumelinu.

Es tev lüdzu, Mārtin brāl,

Dod man vienu ceḷa zirgu. 30219
Martin hat einen vollen Stall dim. mit grauen, braumen Rossen dim.. Ich bitte dich, Bruder Martin, gib mir ein Pferd auf den Weg.

Dieses Liedes zweite Hälfte entspricht unserem 48. Von Ūsiņš aber kann man bekanntlich auch ein Pferd kaufen (61) oder tauschen (18), also nicht nur erbitten, wie vom hl. Martin.

Eine bezeichnende Übereinstimmung der Texte über Ūsinš und den hl. Martin findet sich in denen, die vom Hahnenopfer sprechen. In unseren Liedern 27-30 und 60 ist die Rede vom Hahnenopfer mit der Motivierung, man erhoffe sich davon verschiedene Äußerungen seines Wohlwollens. Alle diese Hoffnungen aber drehen sich darum, daß die Pferde gut sein mögen. Daneben findet sich vereinzelt auch eine Erweiterung seiner Fürsorge für andere Haustiere. Die Texte mit dem Hahnenopfer für hl. Martin sind noch zahlreicher, als die mit dem Opfer für Ūsiňš oder für hl. Georg. Besonders verbreitet ist 30218 , das in $11 \mathrm{~V}$. bekannt ist:

Mãrtinam gaili kāvr

Sarkanām kājiñuam,

Lai guntinas nevajaga,

Kumelinus barojot. 30218

Devu, devu Mārtinam

Ko es biju solijusi;

Melnu vistu kankarainu

Ar visiem nadziniem. 30211
Martin schlachtete ich einen Hahn mit roten Füßen dim., damit man kein Feuer braucht, um die Rosse dim. zu füttern.

Ich gab, ich gab Martin, was ich versprochen hatte: ein schwarzes, zottiges Huhn mit allen Krallen dim.

Vgl. auch 30215, 30216 mit 11 V. (darunter 5 mit Ūsiņš, die 28 entsprechen), 30217.

$\mathrm{Zu}$ den beiden obigen Texten und deren Varianten ist anzumerken, daß deren Inhalt mit dem der zuvor erörterten Texte über Ūsiņš und hl. Georg übereinstimmen: im ersten Teil wird das Opfer erwähnt und in der zweiten Hälfte die Wohltat, die man vom hl. Martin erwartet. Der einzige Unter-

1 Als ein solches läßt sich 3021,2 nicht auffassen, wie die 1. V. desselben Liedes beweist. 
schied findet sich in 30218, nämlich daß die roten Beine des geopferten Hahnes gewissermaßen das Licht, ,beim Fütterm der Pferde" ersetzen, offensichtlich im Stall. In den Ūsinšs-Texten ist an deren Stelle von gelben Beinen die Rede.

Der hl. Martin hat, ebenso wie Ūsins̆, sein Wohlwollen auch auf andere Haustiere ausgedehnt, konkret auf die Kühe:

Märtinami vĩzes pinu, I auklinas darināju, Mārtiňš manas govis gana

Šo garo vasarinu. $\quad 30220$
Ich flocht Martin Bastschuhe, und machte [ihm] Schnüre dim., Martin hütet meine Kühe diesen langen Sommer dim.

Vgl. auch 30221 .

Es mag ganz erstaunlich erscheinen, daß ein christlicher Heiliger im Bischofsrang zum lettischen Kuhhirten werden konnte. Das braucht aber nicht zu überraschen, denn in solcher Funktion erscheinen Personen mit noch höherer Dignität - Jesus Christus als Kuhhirte und die hl. Maria als Melkerin der Kähe und als Schererin von Schafen ${ }^{\underline{x}}$. Am interessantesten ist die Tatsache, daß der hl. Martin dargestellt wird, nicht im würdigen Bischofsgewand, sondern mit der der ärmlichen Stellung des Hirten entsprechenden Fußbekleidung in vĩzes ar auklām 'Bastschuhen mit Schnur'. Bei Usinšs und hl. Georg gibt es derartige Angaben nicht, weder daß sie sich direkt an der Viehhütung beteiligt, noch daß sie derartiges Schuhzeug getragen hätten. Ein Text geht noch weiter und läßt dem hl. Martin ein Huhnopfern, damit die Kühe gut gedeihen:

Cekulainu vistu kāvu

Mōrtenüšsu vakarā,

Lai aug man raibas govis

Kā vistinas celeulainas. 30232
Ein geschopftes Huhn schlachtete ich am Martins dim. Abend, damit mir bunte Kühe wachsen wie beschopfte Hühner dim.

1 Cf. Biezais 1955, 255.

2 Nach ME 4, 650: ,, $\bar{\imath} z e$ [...] der Bastschuh [...] eine aus Lindenborke oder Weiden. rinde geflochtene Fußbekleidung." Dort macht Endzelins weitere Hinweise auf li. viežti und av. ni-vaēz und auch auf weitere Literatur Thomsen 244; Persson 1912, 465; Leskien 600 u. a. Scheftelowitz 1927, 249 s.: ,Walde, o. XXXIV 518 verbindet fälsch. lich abg. vęzati mit lit. wyzà ,geflochtener Schuh, Bastschuh", doch geht das lit. Wort auf idg. *veỉĝ zurück, aw. ni-vā̄z, ,sich anheften, sich anbinden", arm. vēž ,,coperta“", vižak, ,Vorhang, Decke", lit. wijžti ",Sandalen flechten", lett. wifêt ,durchhecheln." Aukla, aukliņa dim. nach ME, 1, 221, ,eine aus Flachs oder Hanf gedrehte Schnur, dünne Leine. [...] li. auklẽ 'Fußbinde', apr. auclo, 'Halfter', lat. sub-ucula und Hin. weise auf Persson 1912, 650 und id. 1918, 128:" [...] lit. auklẽ 'Fußbinde', lett. cuikla 'Pastelschnur, Schnur um etwas festzubinden', preuss. anclo 'Halfter', lit. aũtas 'Fußlappen', lett. auts 'Tuch, Binde', galdauts 'Tischtuch, prëlssch-auts 'Schürze' usw.; arm. z-aud 'Band', $y$-aud 'Band, Glied, Gelenk, aud 'Schuh' (aus au-dh-)."

Derartiges Schuhwerk ist beispielsweise auch in mehreren ethnografischen Arbeiten 
Dieser Text steht freilich allein, und es scheint, daß er durch Kontamination mit den bekannten Vorstellungen über die Opfer an Üsingš entstanden ist. Hier ist daran zu erinnern, da $\mathrm{B}$, wenn ein solches Opfer tatsächlich gebracht worden wäre, es eher der Laima gegolten hat, wie wir es in einem der epischen Texte gelesen haben.

Ein noch merkwürdigeres Bild bieten Texte, in denen Usiņš und ein christlicher Heiliger nebeneinander genannt werden. Wir erörterten schon das Lied 46, in dem Ūsinsš und hl. Georg nebeneinander erscheinen. Die Verwirrung ist in einem anderen der auch schon behandelten Texte (68) noch größer. Dort heißt es, man opfere dem hl. Martin einen Hahn mit neun Schöpfen, aber der Geber des Pferdes im Ergebnis eines solchen Opfers ist dennoch Ūsiņš. Dieser Text bestätigt deren Identität. Freilich haben diese Texte Zufallcharakter. Aus dem Vergleich der Texte über Ūsinšs und hl. Martin erhellt dasselbe, wie aus denen über Ūsinš und hl. Georg. Auch hl. Martin erscheint in der gleichen Funktion, nämlich als Patron der Pferde. Ihm wird gleichermaßen das Hahnenopfer gebracht, damit er die Pferde versorge. Nur in einigen kleinen, aber bezeichnenden Einzelheiten erscheint hl. Martin nicht. So bei der nächtlichen Hütung. In den Texten wird nicht gesagt, daß er zur nächtlichen Hütung reite, was hl. Georg immerhin tut. Das erklärt sich daraus, daß um Martini die nächtliche Hütung längst ausgelaufen ist. Mit hl. Martin wird auch kein Opfermahl verbunden. Er wird zwar als guter und reicher Mann gerühmt, der zur rechten Zeit (,im Herbst, im Frühling) (30223) mit Brot erscheint, er ,schlachtet auch am Abend einen Ochsen" (30225), d. i. am Martinsabend. Aber weder der eine noch der andere Text erwähnen ein dem hl. Martin geweihtes Mahl, sein Name wird offenbar blob in kalendarischem Sinne gebraucht. Das zeigt deutlich auch:

2. $\bar{U} \sin \bar{a}, M \bar{a} r t i n \bar{a}$

Saldu dara alutin,;

Mārtiñ̌s miežus audzināja,

$\bar{U}_{\text {sing }}$ labus kumelinus.
Zur Usingzeit, zu Martini

Braut man süßes Bier;

Martin ließ Gerste wachsen

Ưsinš gute Rößlein. A2

Hier darf ein Text nicht übergangen werden, der in einer Kleinigkeit zeigt, daß Usinş und der hl. Martin jeder einer anderen, ihrer eigenen Welt, angehören:
50. Atnāca $\bar{U} \sin , \bar{s}$ Atritināja, Uzkāara mēteli

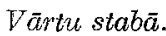

\author{
Der Ūsinš kam, \\ Er rollte herbei, \\ Hing seinen Mantel \\ An den Pfosten des Tores. A 60
}

abgebildet. Cf. Etnogrāfija Abb. 39, 1; 40, 1; 41; Dumpe, Linda, Abb. 41, 44, 45̃. Eine gute Abbildung bei Bielenstein, Martha 147. 
Mārtiňš atrābināja,

Aizrz̈bināja,

Pakāra mēteli

Zobina galā. 30222
Martin dröhnte her, dröhnte davon, hing den Mantel auf die Spitze des Schwertes.

Der erste Text spricht von Ūsinšs, der ,herangerollt" ist und danach seinen Mantel an den Pfortenpfosten gehängt hat. Der andere spricht von hl. Martin, der ",herangedröhnt" ist und seinen Mantel auf die Schwertspitze gehängt hat. Zum zweiten Text ist anzumerken, daß er jedenfalls unter dem direkten Einfluß anderer Texte entstanden ist, sei es nun unter dem des Ūsinšs-Textes, in dem zufällig oder bewußt atritinäja 'herangefahren' durch atribināja 'herangedröhnt' ersetzt wurde, sei es unter dem eines anderen. Der Dröhner par excellence ist Pērkons und ein solcher Text mag sich ursprünglich auf diesen bezogen haben ${ }^{1}$. Welcher Art der ursprüngliche Text gewesen ist, ist nicht das Wichtigste. Wichtig ist, daß beide im Mantel erscheinen, der eine den am Pfortenpfosten aufhängt, der andere aber an der Schwertspitze. Gerade dies zeigt beider wahre Natur und ihre Zugehörigkeit zu verschiedenen Welten. Der Heilige der erobernden Ritter trägt des Ritters Ehrenzeichen - das Schwert. Ein solches hatte der andere Reiter - Usinšs - nicht. Vielleicht war sein Ehrenzeichen eine volle Hand Pferdezäume ${ }^{2}$.

4.2.3.3. Außer diesen beiden, hl. Georg und hl. Martin, erscheint noch ein dritter christlicher Reiter in der Funktion des Ūsiňš. Das ist der hl. Michael, lett. Mikelis, Mikiklïtis dim., auch Mikals, Miklävs und andere Mundartformen. Vor allem stellt dieser sich würdig an die Seite von hl. Georg als Versorger der Pferde und als Zeuge beim Pferdehandel. Er nimmt auch an dem dem Handel folgenden Mahle teil (30052). Es wiederholen sich hier die von hl. Georg und hl. Martin her bekannten Motive: auch er ,richtete sich hoch auf hinter meinem Pferdestall". Vgl. 51574 oder auch:

Mīkals stāv aiz vārtiem,

Santa svärki magurā.

Nãc, Mīkal, kambarī,

Sédies galda galiñā. $\quad 30059,1$
Michael steht vor dem Tor, mit einem Samtrock bekleidet. Komm, Michael, in die Kammer, setz dich ans Ende dim. des Tisches.

In diesen Liedern erscheinen in anderen Varianten hl. Georg (30059) und Üsiňs (2. V., entspricht 1). Darin sind Spuren des Hintergrundes zu erkennen, aus dem hl. Michael kommt. Er trägt einen Samtrock ${ }^{3}$. In Samt

1 Cf. Biezais 1972, $104 \mathrm{~s}$.

a Daß der hl. Martin, wie der hl. Georg, in die lettische Volksüberlieferung eindringen konnte, ist verständlich. ,Name and Leg. des hl. Marin sind mit den ersten Anfangen der Geschichte des Christentums in Livland und der Missionstätigkeit Bif. Meinhards verknüpft" (Bruiningk 479).

s Cf. Zēvers 104: ,sañts, sañts 'Samt'<mnd. sammet." 
gekleidete Heilige waren eine gewöhnliche Erscheinung in der christlichen Kirche. Aber der für die Annäherung von hl. Michael an Ūsiṇš ein bezeichnender Text ist:

Miklavā gaili kāeru

Deviniem nadziniem;

Miklavā zirgu pirlou

Deviniem iemauktiem. 30227
Zu Michaeli schlachtete ich einen Hahn mit neun Krallen dim.; zu Michaeli kaufte ich ein Pferd mit neun Zäumen.

Dennoch zeigt dieser Text einen bemerkenswerten Unterschied. Es heißt in ihm, zu Michaeli sei ein Hahn geschlachtet worden. Das verbindet ihn unmißverständlich mit der Gruppe der Ūsinš-Lieder. In der zweiten Hälfte der Strophe aber fehlt die übliche Fortsetzung, die Motivierung nämlich, wozu das getan wird. Zudem fehlt auch das Wesentliche, der Charakter als Opfer. Statt dessen wird gesagt, der Sänger kaufe gerade an diesem Tage ein Pferd. Er selbst ist der Aktive, er bestimmt seine Handlung. Die letzte Zeile aber läßt erkennen, daß es sich doch um ein besonderes Pferd handelt. Der Text enthält Elemente der uns bekannten Gruppe der Ūiņš-Lieder, aber es fehlt die Motivierung des Opfers. Mit anderen Worten, darin ist bloß noch ein blasser Widerschein des ursprünglichen Gedankens.

Die Texte mit dem hl. Michael sind viel weniger zahlreich, als die ähnlichen mit hl. Georg und hl. Martin. Das läßt sich in diesem Falle nicht damit begründen, daß solche Texte in geringerem Ausmaß aufgeschrieben und eingesandt worden wären. Wenn das so wäre, müßte es in gleichem Maße auch für die Texte mit hl. Georg und hl. Martin gelten. Deshalb ist anzunehmen, daß hl. Michael in geringerem Maße in diese Texte eingedrungen ist ${ }^{1}$. Es ist eine in der christlichen Ikono- und Hagiographie bekannte Erscheinung, das der hl. Georg und der hl. Michael oft miteinander verschmelzen, worauf schon öfter hingewiesen worden ist². So hat das auch in der lettischen Volksüberlieferung erfolgen können.

4.2.3.4. Ūsiņš erscheint aber auch in weiterer christlicher Gesellschaft. Als wir die epischen Quellen behandelten, bemerkten wir, daß das dem Ūsiṇš geweihte Mahl auch in andere Zeiten verlegt wird, etwa auf Pfingsten oder gar auf Johanni. Das wird auch durch einige, insgesamt 3 Dainas bestätigt:

Jānäts jāja piegul̄a

Deviniem kumeliem;

Es tecēju vārtu vērti,

Man iedeva devito. 30058

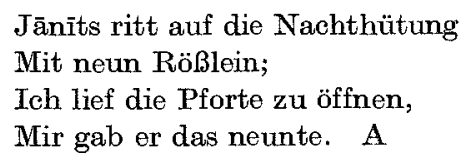

1 Über den geringen Einfluß von Michael in der christlichen Kirche in Lettland s. Bruiningk $493 \mathrm{~s}$.

2 Über die Verschmelzung der Funktionen dieser beiden Heiligen hat sich unter Hinweis auf geistesgeschichtliche und geographische Motive besonders Krefting, $13 \mathrm{~s}$, $34 \mathrm{~s}$, geäußert. 
Dazu ist zu bemerken, daß dieser Text fast wörtlich mit unserem Text 9 übereinstimmt. Es ist nicht anzunehmen, daß Jānis (Johannes) hier infolge des unbestimmten Kalenders eingedrungen ist, oder gar wegen der Unwissenheit oder Unaufmerksamkeit der Sagerin. Das hat andere Motive. In den altlettischen Traditionen ist Jānis ein Reiter mit hervorragender religiöser Bedeutung¹. Ūsinšš und Jānis gehören jeder zur eigenen Gruppe religiöser Vorstellungen, deshalb gibt es so wenig Zeugnisse für ein Nebeneinander von Jānis und Ūsinšs.

Beim Studium der christlichen Gesellschaft des Ūsiņš erweist es sich, daß er im christlichen Kalender seinen Platz neben Martin und Michael hat $(2,3,4,5,6)$. In einigen, anscheinend späteren Schöpfungen unterhält er sich mit Tenis (hl. Antonius) (34). Als Pferdepatron muß er sich nicht nur einmal der Patronin der Kühe Māṛa bzw. Māršava (hl. Maria) gesellen $(40,64)^{2}$. Einer der ausdrucksstärksten Texte, die seine christliche Gesellschaft bezeugen, ist 77 :

77. Zirgu $\bar{U} \operatorname{sins}$, Govu Māršaviña,

Kazu Barbina,

Cüku Teniss,

Aitu Annūte,

Telu Urbāns,

Sargat manus kustonī̌̌us

No sērgām, no vilkiem! 29202
Pferde-Ūsinšs, Kühe-Māršavina, Ziegen-Barbina, Schweine-Teniss, SchafeAnnite, Kälber-Urbāns, hätet meine Tierchen vor Seuchen, vor Wölfen!

Einige der hier exörterten und genannten Texte belegen bloß die Entstehung einer Tradition in der Ūsinš anderen christlichen Patronen ebenbürtig geworden und in den christlichen Kalender gelangt ist.

4.2.3.5. Wirft man einen Blick zurück auf die Beurteilung verschiedener Anschauungen, die das Wesen des Üsinšs mit fremden Einflüssen zu erklären versuchen, zeichnet sich ein recht deutliches Bild ab. Zuerst sind alle Versuche abzulehnen, den Namen Ūsiņš mit der ide. Wurzel *aues zu verbinden und wie auch die semantische Bedeutung desselben davon abzuleiten. Damit entfällt auch dessen Verknüpfung mit ähnlichen $\mathbb{E r}$ scheinungen (Ușas, Ausma, "Ews, Aurora u. a.). Das bedeutet, daß hierin Auniņš, Volteris, Ķiparts, Schröder u. a. geirrt haben. Es erklärt sich dies aus derzeitigem großem Vertrauen auf etymologische Beziehungen und deren Bedeutung für die Bestimmung des Wesens von Gottheiten.

Zweitens ist die von Endzelins selbst korrigierte Ansicht als richtig

1 Darüber kann bei Biezais, 1972, 360 ss, mit umfangreicher Materialanalyse nachgelesen werden.

2 Über die Namen $M a \bar{r} r a$ und $M a \bar{r} s ̌ a v a$ und die Problematik der betreffenden Wesen habe ich mich an anderer Stelle speziell geäußert. Cf. Biezais 1955, $258 \mathrm{ss.}$ 
anzuerkennen, daß der lettische Ūsiņš nicht vom deutschen Husing abzuleiten ist. Offenbar hat in der Anfangszeit der Forschung die phonetische Ähnlichkeit der beiden Wörter zu dieser Ansicht geführt.

Drittens ist die Behauptung unbegründet, Usiṇš habe seinen Namen and seinen religiösen Gehalt von den Russen bekommen, wie das ihrerzeit Endzelins und Šmits behaupteten. Im vorigen Abschnitt haben wir schon die Motive aufgezeigt, die die Sprachwissenschaftler zwingen, die Entlehnung des Namens Ūsinš vom russischen Усень zu verwerfen. So erklärt sich auch, daß die russische Volksüberlieferung kein personifiziertes Wesen kennt, das den Vorstellungen über Ūsiņš entspräche, oder dessen Funktionen in der lettischen Religion beeinflußt haben könnte. Die Anschauung ist ihrerzeit unter dem Einfluß der Versuche, etymologische Beziehungen herzustellen, entstanden. Finige Forscher haben versucht, die vermuteten etymologischen Beziehungen durch angebliche gemeinsame Züge der Überlieferung zu stützen. Uberprüt man das, erweist es sich, daß es dafür der Grundlage ermangelt.

Viertens muß anerkannt werden, daB die Quellen von sehr weitgehender Identifizierung von Usinšs mit hl. Georg, hl. Martin und hl. Michael zeugen. Das Folklorematerial zeigt, daß er auch in Gesellschaft anderer christlicher Heiliger erscheint und sich in deren Funktionen teilt. Der lettische religiöse Synkretismus zeigt offenbar im Falle des Ūsinšs die Verschmelzung vorchristlicher und christlicher Traditionen. Man hätte erwarten können, daß aus der engen Beziehung des Usinš vor allem mit hl. Georg auch zusätzliche Klarheit auf die eventuelle Beziehung zwischen seinem Namen und $\vec{u} s a s$ Schnurrbart hätte fallen können. Müthel hat nicht gehofft, für die Erhärtung dieser Beziehung Unterstützung von Seiten eines christlichen Heiligen zu finden, aber er spricht hypothetisch von einem heidnischen Götzen mit Schnurrbart. Ebensogut kann vermutet werden, ein christlicher Heiliger mit Schnurrbart hätte den Impuls, für den Namen des Usiṇš bewirken können. Die obige Erörterung zeigt, daß man derartiges von erwähnten Heiligen erwarten könnte. Der älteste Stiertöter Mithra und der Drachentöter hl. Georg zu Fuß und zu Pferde werden als jünglinghafte Kämpfer vorgestellt ${ }^{1}$ Es ist auch keine lokale Tradition über einen schnurrbärtigen Heiligen bekannt. Deshalb ist nicht anzunehmen, daß aus dieser

1 Das Auftreten eines bärtigen hl. Georg in der christlichen Kunst ist eine sehr späte und dazu vereinzelte Erscheinung, worauf Taube, 203, hingewiesen hat: ,In den letzten Jahrzehnten des Trecento wird Georg in der Regel bärtig dargestellt. Die uns bekannt gewordenen Darstellungen sind alle aus Oberitalien. Meist hat er eine Barttracht, wie sie damals auch von jungen Leuten getragen wurde, und jugendliche Züge. Auf dem Drachenkampf in San Salvatore und auf den Fresken der Kapelle zu Padua ist der Heilige kein Jüngling mehr, mit Vollbart und dunkel. Sonst bleibt er blond." Cf. auch Küppers, 8, besonders die Bild.Beilagen. 
Richtung eine Unterstützung für die Anschauung zu erwarten wäre, Ūsinšs hätte seinen Namen unter Einfluß des Schnurrbarts eines Heiligen erhalten.

Am Schluß des Überblicks über die verschiedenen Gedankengänge und empfohlenen Lösungen für das Usiņ̃̌-Problem sind wir zu dem Punkt gelangt, wo zu fragen ist, ob es dafür überhaupt eine Lösung gibt und welche das wäre. Mit anderen Worten, es stellt sich zum wiederholten Male die Frage - wer ist Ūsiņšs? Im nächsten Abschnitt soll versucht werden, diese Frage zu beantworten. 